\title{
LIS: Localization based on an intelligent distributed fuzzy system applied to a WSN
}

\author{
D.F. Larios, J. Barbancho, F.J. Molina, C. León \\ Department of Electronic Technology, University of Seville, Escuela Politécnica Superior, C/Virgen de \\ África $\mathrm{S} / \mathrm{N}$, Seville, Spain
}

Keywords:

Fuzzy system
WSN
Localization
RSSI
Centroid

$\mathrm{CL}$
The localization of the sensor nodes is a fundamental problem in wireless sensor networks There are a lot of different kinds of solutions in the literature. Some of them use external devices like GPS, while others use special hardware or implicit parameters in wireless communications.

In applications like wildlife localization in a natural environment, where the power available and the weight are big restrictions, the use of hungry energy devices like GPS or hardware that add extra weight like mobile directional antenna is not a good solution.

Due to these reasons it would be better to use the localization's implicit characteristics in communications, such as connectivity, number of hops or RSSI. The measurement related to these parameters are currently integrated in most radio devices. These measurement techniques are based on the beacons' transmissions between the devices.

In the current study, a novel tracking distributed method, called LIS, for localization of the sensor nodes using moving devices in a network of static nodes, which have no additional hardware requirements is proposed.

The position is obtained with the combination of two algorithms; one based on a local node using a fuzzy system to obtain a partial solution and the other based on a centralized method which merges all the partial solutions. The centralized algorithm is based on the calculation of the centroid of the partial solutions.

Advantages of using fuzzy system versus the classical Centroid Localization (CL) algorithm without fuzzy preprocessing are compared with an ad hoc simulator made for testing localization algorithms.

With this simulator, it is demonstrated that the proposed method obtains less localization errors and better accuracy than the centroid algorithm.

\section{Introduction}

A Wireless Sensor Network (WSN) consists of a lot of small devices deployed in a physical environment. Every device, called a node (Fig. 1), has special capabilities, such as communicating with its neighbors, sensing and data storage and processing. The nodes can make a mesh network of devices in such a way that they can collaborate

Corresponding author. Tel.: +3495455 2838 .

E-mail addresses: dflarios@dte.us.es (D.F. Larios), jbarbancho@us.es (J.

Barbancho), fjmolina@us.es (F.J. Molina), cleon@us.es (C. León). amongst themselves can collaborating amongst themselves. These features permit the implementation of distributed solutions to solve complex problems.

The main components of a node of WSN are a microcontroller, a wireless transceiver, a power source and Input and Output modules ( $\mathrm{I} / \mathrm{O})$ to interact with the environment (Fig. 2).

The microcontroller processes the data and controls the functionality of the other components in the sensor node. Low power microcontrollers are typically used in WSN.

Wireless modules use ISM bands which allow transmissions over some special license-free frequencies with 


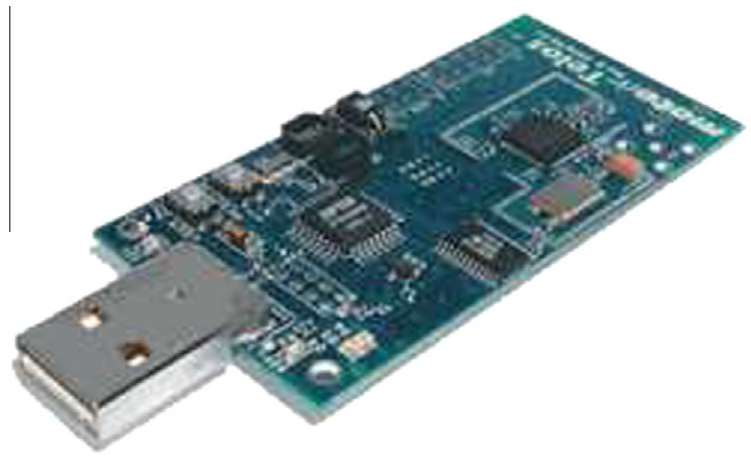

Fig. 1. Example of a WSN node.

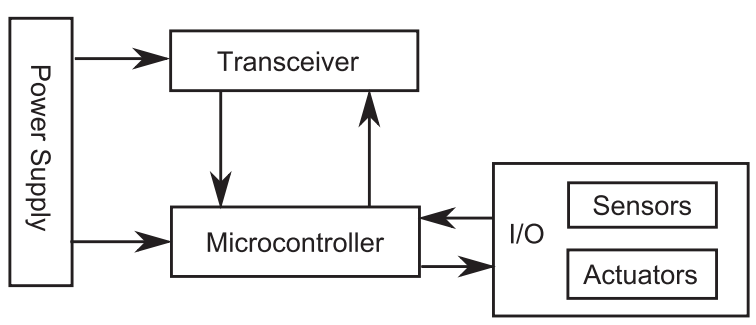

Fig. 2. Architecture of a WSN node.

global availability (like the 173, 433, 868, $915 \mathrm{MHz}$ and $2.4 \mathrm{GHz}$ band). The functionality of both the transmitter and the receiver are combined into a single device known as transceivers. Transceivers for WSN often lack unique identifiers and have low power consumption. Recently, standard protocols, such as IEEE 802.15.4 [1] and ZigBee [2] have specially developed for these devices.

The power source on a WSN is provided by batteries or capacitors [3]. Batteries can be rechargeable and nonrechargeable depending on the application. Solar panels are the typical energy sources for the refill batteries.

Typically, a WSN is formed by very small devices with several restrictions: low power consumption, low weight (especially for mobile devices), low cost, low data storage and processing and low radio range.

Generally, amongst all the node components, the greatest energy consumption is dissipated in the radio transceiver. As an example, the TelosB platform consumes $41 \mathrm{~mW}$ in active mode. The microcontroller consumes only $5 \mathrm{~mW}$ and the remainder power consumption is caused by the radio transceiver that requires $38 \mathrm{~mW}$ in the receiver mode and $35 \mathrm{~mW}$ in transmission [4].

It is important to point out that the power consumption is very high either in transmission or in the reception mode. Therefore, to reduce the power consumption it is necessary to reduce the number of message exchanges, and also stop all the node activity enabling low power modes by switching off the radio transceiver. Therefore, a suitable activity manager with hibernation periods is needed.

Several protocols for hibernation have been proposed [5-9] looking for a reduction in the power consumption. The main problem of these protocols consists in setting the clock synchronization in every node; otherwise a node can send a message when all its neighbors are in idle stage and losing information.

WSN has been widely used in many areas [10], such as environmental monitoring [11] and control [12], healthcare and medical research [13], national defense and military affairs $[14,15]$, etc.

For all of these applications which have mobile or autonomous nodes, it is usually necessary to implement a hibernation mechanism to save power. Moreover, many of these applications require the position knowledge of a significant amount of nodes.

In some of these applications the information gathered from the nodes is not relevant without the knowledge of the associated position, for example, describing a system for wildfire tracking based [16] on the determination of specific situations (temperatures, humidity, wind direction, etc.). The position of the sensors that describes these situations should be known.

In other applications the information required is the position itself, for example, in vehicle tracking [17].

Thus, it can be seen why localization is one of the crucial issues in wireless sensor networks research. In a lot of cases it is impossible to use specific localization devices, like a GPS, because these devices have huge energy consumption and reduce autonomy. In other applications it is necessary to have nodes in the interior of buildings, where GPS technology does not work correctly [18].

The main contribution of the current study is the presentation of a novel localization algorithm, based on fuzzy logic processing, and implemented over small wireless sensor nodes. As the devices run a fuzzy logic algorithm for processing the information, it was considered that the devices were provided with some kind of intelligence. Thus, the system has been named as LIS, i.e. Localization based on Intelligent System.

The above mentioned localization algorithm is designed specifically for tracking small mobile devices with very low weight, thereby permitting to obtain the position of small animals. Therefore, no additional hardware is used.

The proposed protocol takes into account the necessity to keep the power consumption low. In this sense, the presented algorithm implements a hibernation protocol for the non-anchor nodes that can save power energy on these devices.

The rest of the current study is organized as follows: Section 2 sums up the state of the art about localization. Section 3 describes LIS. The outcome of LIS performance is developed in Section 4. Finally, Section 5 presents the concluding remarks and provides the discussion for future studies.

\section{Localization techniques}

In localization applications, there are two types of nodes:

- Anchor Nodes: situated on fixed and known positions.

- Non-Anchor Nodes (tags): nodes with unknown position. These nodes are usually called tags. 
For many application, it is important to consider that anchor nodes are less energy constrained than non-anchor nodes. This is because in anchor nodes systems to recharge the batteries, such as solar panels, can be used. For many applications (as in tracking small animals) these recharge systems can not be used in non-anchor nodes, due to the constraints of maximum size and weight.

Fig. 3 shows the typical WSN used for localization proposes. The essence of localization consists in obtaining the position of the non-anchor nodes, using the information provided by the anchor nodes.

The localization research over WSN is focused on the following points: determining a new localization algorithm, modifying the proposed solution in order to improve accuracy or testing classical methods [19]. Testing can be focused on specific applications, such as indoor uses $[20,21]$, or centered on specific characteristics, such as energy efficiency [22].

Localization algorithms presented in the literature can be classified into two categories, as given below:

\subsection{Range-based techniques}

These techniques estimate, point-to-point, the distance between each pair of nodes.

With this information and using techniques, such as multilateration [23], triangulation or other methods, the absolute position of the non-anchor nodes can be estimated. The most common ones are Received Signal Strength Indication (RSSI) [24], Time Of Arrival (TOA) [25], Time Difference Of Arrival (TDOA) [26] and Angle Of Arrival (AOA) [27,28].

Nowadays, new algorithms continue to appear based on these classical methods for the improvement of accuracy, for example, based on AOA [29-33], based on TOA $[34,35]$ and based on TDOA [36,37].

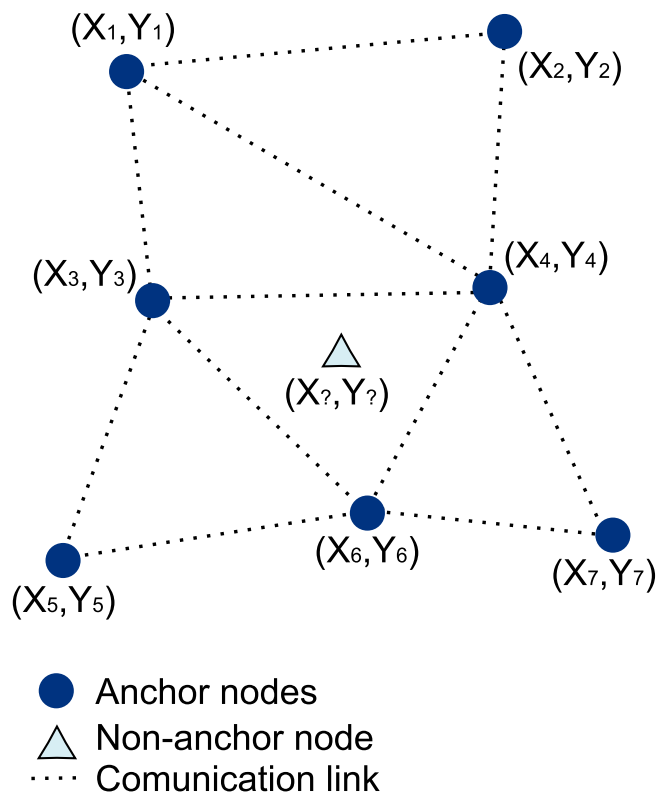

Fig. 3. Example of WSN for localization.
Other authors propose new range based algorithms, like $\mathrm{Su}$ [38] that uses a likelihood calculation for determining the distance.

In general, the range-based ones offer good accuracy, but additional hardware is often needed. Therefore, the weight, the cost and the power consumption of the node devices increase and make these techniques unsuitable for the proposed application. RSSI range-based techniques are an exception to this because most of the current transceivers provide this measurement by default. However, RSSI techniques are very sensitive to noise and interferences. The problem of the RSSI measurement is described in Section 4.1.

These RSSI techniques require calibration and a model of the environment. Moreover, the calibration could change according to the environmental conditions. Nowadays, the determination of how to improve the accuracy of the RSSI techniques is an important research area [39-41].

In order to avoid the RSSI problems, the proposed method uses a fuzzy RSSI model, instead of a mathematical model, as described in Section 3.

\subsection{Range-free techniques}

In the range-free localization algorithms the position of the non-anchor nodes is obtained from the beacons exchange among nodes (anchor and non-anchor nodes). Beacons can contain different information, such as:

- Radio coverage membership: An anchor node detects whether a non-anchor node is in its radio coverage. Using this information, the system can estimate the non-anchor position as a function of the intersection of the coverage areas of every anchor node that are in its radio coverage. Fig. 4a shows an example for the intersection of the coverage areas between the two anchor nodes.

- Number of hops to an anchor-node: If there is no connectivity with an anchor node, a non-anchor node can estimate its position knowing the number of hops to every anchor node. An example of that is represented in Fig. 4b. Node B is at a distance of two hops to anchor node one, three hops to anchor node two and two hops

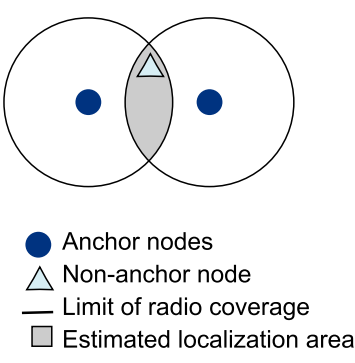

(a)

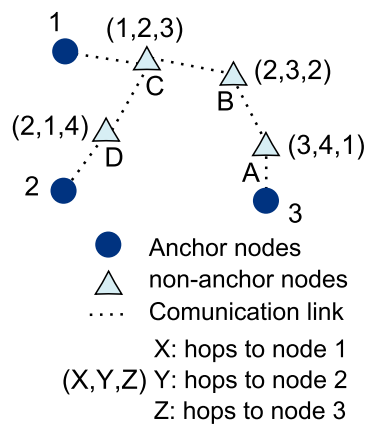

(b)
Fig. 4. Range-free techniques: (a) Radio coverage membership. (b) Number of hops. 


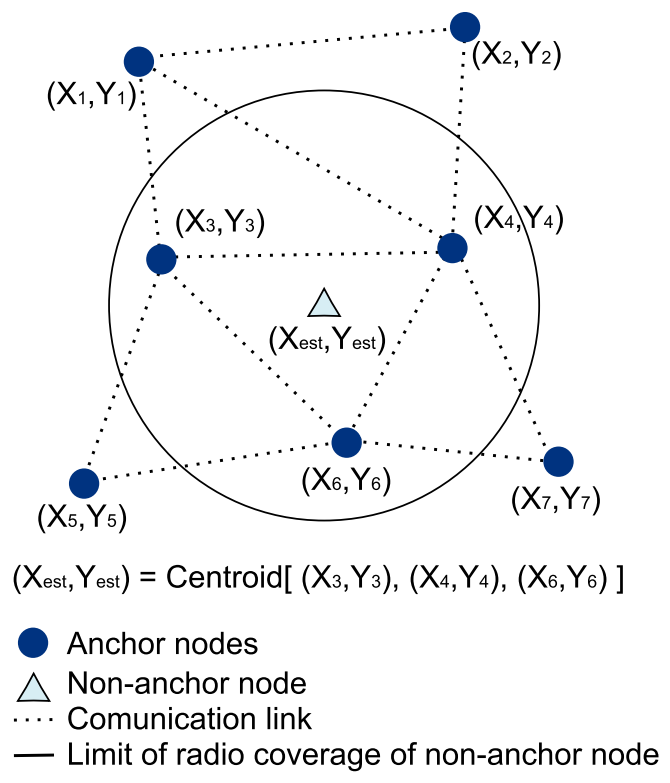

Fig. 5. CL algorithm.

to anchor node three. According to this information and considering the mean distance between the nodes, the absolute position can be calculated applying algorithms, such as triangulation.

The most common range-free techniques are Centroid (CL) [42], DV-Hop [43], Convex [44], APIT [45], etc.

$\mathrm{CL}$ is based on the estimation of the position of a nonanchor node by the centroid of the position of all of its anchor node neighbors. Fig. 5 represents an example where a beacon of non-anchor node is received by anchor nodes 3,4 and 6 , thereby obtaining the estimated position as the centroid of the coordinates of these nodes.

Nowadays, modifications of the $\mathrm{CL}$ algorithm are an important area of research. Many authors continue proposing modifications that offer better accuracy by weight obtained with RSSI [46] or with the Link Quality Indicator (LQI) [47]. Some approaches have focused on the reduction of energy consumption. Behnke [48], proposed a modified $\mathrm{CL}$ algorithm without the use of complex mathematical operations for low resources microcontroller like the square root. Other approaches have studied the specifications of the CL algorithm in order to determine the areas where it offers bigger errors [49].

New range-free algorithms continue to appear in the literature [50]. Some of them are focused on determining the new aspects of the networks that can give information about the localization, such as the intersections of a simplified coverage area [51], or modifying the power transmission of the beacons in order to obtain the smallest area where it is more probable to locate the non-anchor node [52]. Others are focused on improving any kind of a characteristic, such as energy efficiency $[53,54]$.

Many range-free algorithms, such as centroid and most of its variations, are fully centralized algorithms, i.e. all the information, useful or not, is sent to a Base Station, where the position of the non-anchor nodes is obtained. This feature constitutes a disadvantage, because it requires the delivery of a large amount of messages to the Base Station, which would waste energy. This is especially important in wide networks.

Against this focus, the rest of the authors propose the use of fully distributed algorithms, typically executed over the non-anchor nodes. However it constitutes a disadvantage because the non-anchor nodes must maintain the radio transceiver during long periods of time. These devices have the highest energy constraint in the network. In other words, it is important to remember that for this tracking application, the Base Station needs to know the position of the non-anchor nodes. Using a fully distributed algorithm requires the delivery of the additional messages from the non-anchor nodes to the Base Station, thereby losing a part of the advantages of distributed algorithms.

The proposed algorithm acts in a different manner. It uses two algorithms looking forward to obtain a part of the advantages of these two focuses: a distributed algorithm, but only executed over the anchor nodes and a centralized algorithm to obtain the final position by the Base Station.

\section{LIS algorithm}

Despite the fact that the range-free and range-based techniques have been extensively studied, nowadays there are some aspects that continue to be a challenge:

- The use of additional hardware or lots of beacons to increase power consumption.

- Fully centralized processing (i.e. on Base Stations) requires a large amount of messages. Conversely, processing in the tags' nodes reduces the battery of these devices significantly.

- Scalability. Many localization algorithms are hard to extend to big sensor networks.

LIS has been especially designed to phase out all of the above mentioned problems. As a result, the proposed algorithm is scalable and the power consumption and network autonomy are optimized. As usual in a tracking system, the non-anchor nodes in LIS do not need to know their location. In this case, only the Base Station wants to know it.

The proposed localization algorithm is a range-free tracking technique that uses RSSI. However, instead of using a mathematical model, LIS proposes a fuzzy-logicbased system to derive the distance from the RSSI level.

Fuzzy logic is a form of multi-valued logic; it deals with reasoning that is approximate rather than fixed and exact. To do this, Fuzzy logic uses variables that have a truth value in a [0-1] range, commonly called membership degree. A fuzzy system offers many advantages, such as its robustness in noisy and complex scenarios.

The use of computational intelligence in localization is not a novel idea, as could be seen in previous works, such as [55] that uses probabilistic neuronal networks, [56] that applies a fuzzy system and [57] that uses fuzzy neurons. 


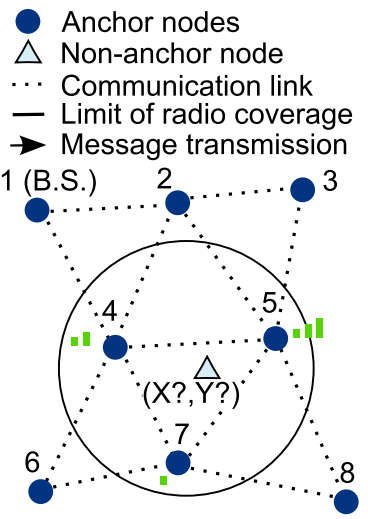

(a)

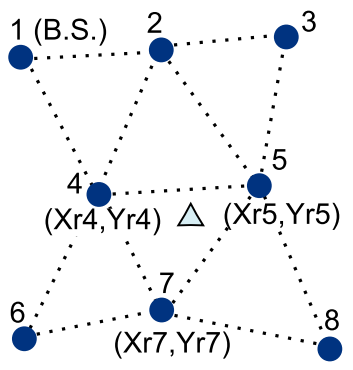

(c)

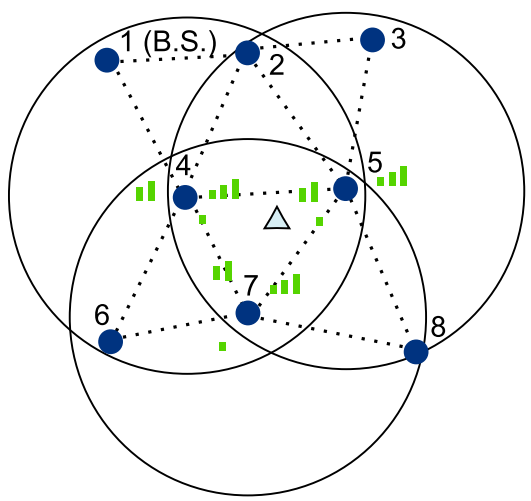

(b)

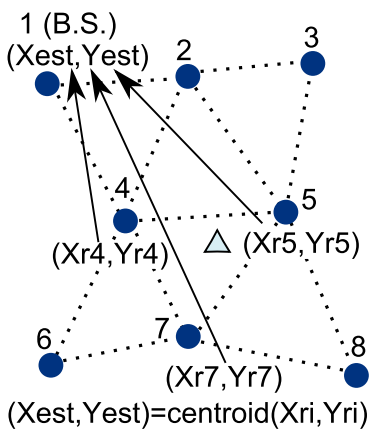

(d)

Fig. 6. Stages of LIS algorithm. (a) S2. (b) S3.A - fuzzyfication. (c) S3.B - Partial positioning (d) S4.

In general, all these are distributed algorithms that execute most part of the localization algorithm over the nonanchor nodes. However, none of them consider the problem of power consumption in the non-anchor nodes. Moreover, the algorithms with Computational Intelligence generally track down the current positions based on the estimated position changes, needing an initialization of the non-anchor nodes. These systems fail if the tags (the animal) go out of the coverage of the WSN, and return into it after a while. As described in Section 5, the authors are currently considering a method to improve the accuracy based on these estimation changes, but without the necessity of an initialization.

\subsection{Network processing stage}

LIS combines: (1) a fuzzy system to estimate (actually to qualify) the distance between the transmitter and the receiver from RSSI measures, (2) a distributed algorithm executed in receiver anchor nodes in order to determine the relative positions to them and filter useless information, and (3) a centralized algorithm to derive the most likely location running at the Base Station.

LIS consists of four stages:

S1: Anchor nodes wait for non-anchor node beacons.

S2: The tag node broadcasts a beacon.
S3: Receiver anchor nodes measure RSSI, and execute both, the fuzzification algorithm and the ubiquitous processing for relative and partial positioning.

S4: Anchor nodes send partial solutions to the Base Station, where the location is finally determined.

Fig. 6 illustrates these stages. When a non-anchor node broadcasts a beacon or any other sort of message, the localization process starts (Fig. 6a). Just at that moment the receiver anchor nodes participate in the process. The rest of the nodes can switch off the radio transceiver or hold it in a low power state.

The beacon frequency can be determined by the application, and can be modified by external conditions, such as the remaining battery in the non-anchor node, a diary schedule or with information registered by external sensor, such as an accelerometer. In this way, the battery autonomy can be improved.

\subsubsection{Distributed processing}

LIS uses the measures of RSSI of a non-anchor node received by an anchor node and its neighbors anchors to determine the area where the non-anchor node could be located. This algorithm is based on a fuzzy system distributed on every anchor node of the network (Fig. 7).

According to the algorithm stages, once an anchor node receives a beacon, it estimates the position of the non-an- 


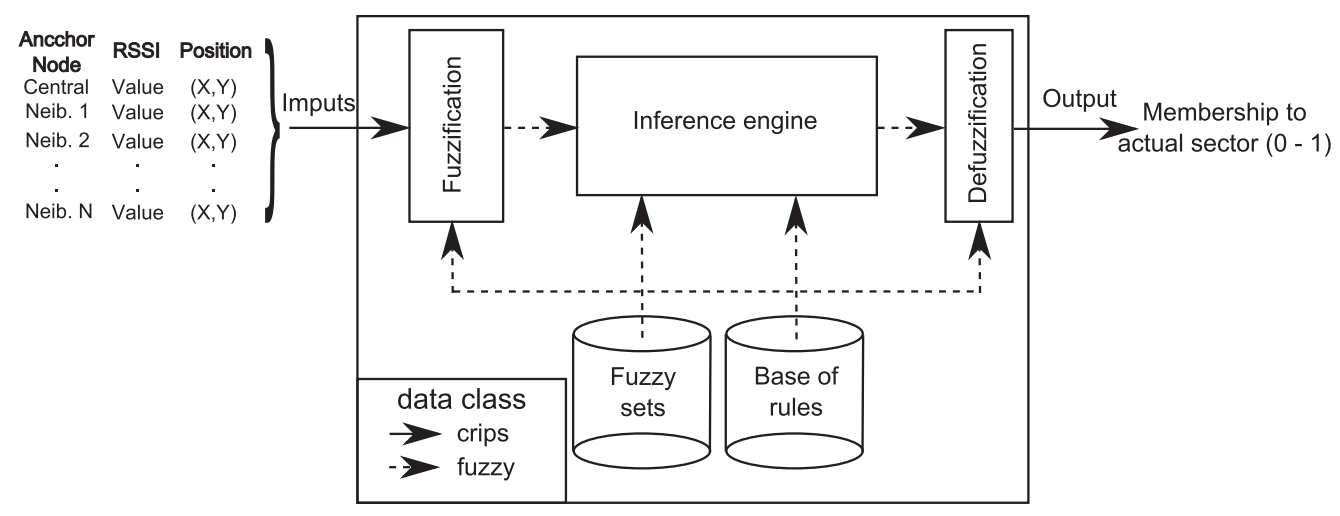

Fig. 7. Inference fuzzy system.

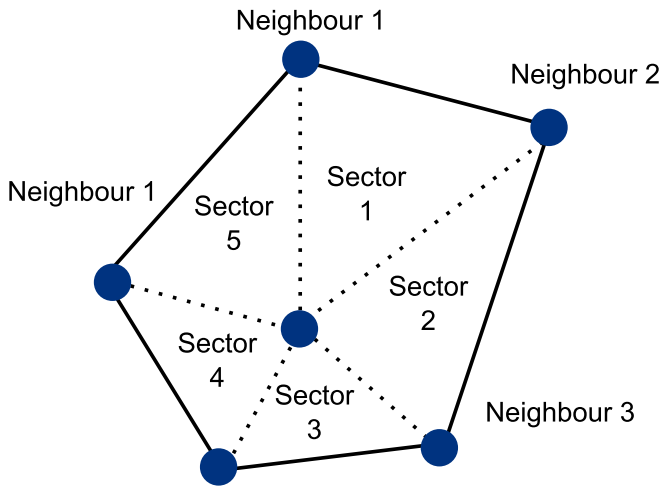

Neighbour 4

\section{Anchor nodes .... Comunication link}

Fig. 8. Example of node with 5 neighbors.

chor nodes. The localization algorithm has been designed to distribute the computation consumption over the network. The area where the non-anchor node could be localized with a certain probability is called the Representative Area. A "sector" is the minimum area formed by three anchor-node neighbors. A Representative Area can be made up of one or more sectors. Anchor nodes must execute the distributed fuzzification algorithm for every surrounding sector. Fig. 8 shows an example with five sectors, in which, the fuzzy algorithm is executed five times.

Every anchor node that receives a beacon measures and broadcasts the RSSI level to its anchor neighbors (Fig. 6b). In this way, the closest anchor nodes elaborate a table with the RSSI measured by themselves and their anchor neighbors.

The RSSI table is processed by the fuzzy system to evaluate the Representative Area, irrespective of the number of sectors. This area can be formed by the union of one or more sectors (Fig. 6c). A sector is considered as a part of the Representative Area if its membership degree (i.e. the output of the fuzzy system) is higher than a threshold. This

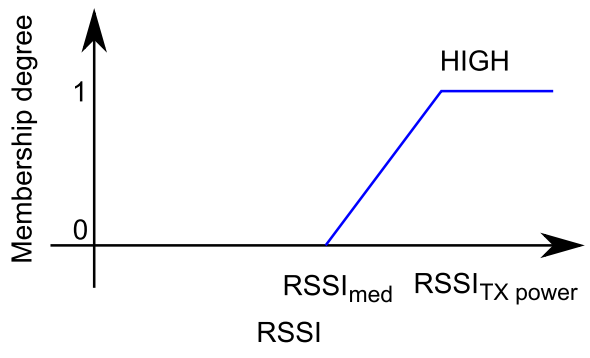

Fig. 9. HIGH fuzzy set.

value is adjusted experimentally. The current simulations show that a threshold of 0.1 manages a good trade-off between the noise immunity and localization performance. The results of the Representative Areas are sent from the anchor nodes to the Base Station to compute the final solution (Fig. 6d).

A Representative Area is empty if it does not contain significant sector, i.e. if the membership degree for all of them is lower than the threshold. In this case, to save energy, the result is discarded and the algorithm will finish until the next beacon arrives. This is especially important in huge networks, where the energy needed for multihop transmissions is high and is a disadvantage of the centralized localization algorithm.

\subsubsection{The fuzzy system inputs}

RSSI tables represent the signal level received in either the local or the neighboring anchor nodes. These RSSI measurements are used as the inputs of the fuzzy system. In these sort of systems, it is necessary to define several fuzzy sets. Every fuzzy set is described as a continuous mathematical function that represents how an input signal belongs to this set. This is called "membership degree" in the literature. This degree is represented in range $[0,1]$, where 0 represents $0 \%$ of membership and 1 represents $100 \%$ of membership to this set. For example, Fig. 9 shows the membership degree of the HIGH fuzzy set, considering the RSSI value as the input signal.

In our system, three fuzzy sets qualify the RSSI as HIGH, MEDIUM and LOW for each input (Fig. 10). 


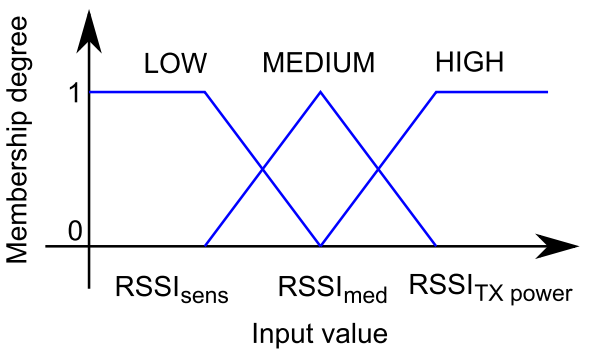

Fig. 10. Sets of the fuzzy inputs.

The LOW RSSI fuzzy set is represented by a trapezoid The maximum membership degree (value 1) is assigned if the power falls bellow the sensibility threshold of the emitter node $\left(R S S I_{\text {sens }}\right)$. As the power increases, the membership degree decreases linearly until it reaches zero at medium RSSI value $\left(R S S I_{m e d}\right)$.

The MEDIUM RSSI fuzzy set is represented by a triangle where the maximum membership degree corresponds to the medium RSSI value ( $\left.R S S I_{m e d}\right)$. The zero membership is reached for the power RSSI values lower than the sensibility threshold or close to the maximum transmission $\left(R S S I_{T X}\right.$ power). In the current study, the medium RSSI value must be computed for every sector using the Friis model equation and assuming the emitter tag is located at the center. This computation only needs to be executed once because the anchor nodes are located at fixed positions.

The use of Friis is a trade-off between the accuracy and the information required. More realistic models require having more initial information of the environment, a priori unknown, such as the position of the obstacles. The Friis approximation simplifies the problems of the saw-tooth of the disturbances with a smooth function. According to a real scenario, this assumption could not be a good approximation to the reality. However, the errors assumed with this approximation are compensated with the noise immunity of LIS, which assumes the disturbances as noise. The authors consider that the use of an in situ calibration, measuring the real received value in the center of the sector would improve the accuracy, but this value would change if the environment changes (i.e. if it is raining or if the grass grows). As mentioned in Section 5, the authors think that measuring the RSSI between the anchor nodes, using the broadcast messages of LIS (it does not increase the total number of messages), would be a better option, because the calibration would be updated at runtime if the environment changes. Currently the authors are evaluating this option.

Fuzzy set for HIGH RSSI values is a trapezoid with a lineal increasing from 0 to 1 for the RSSI power values ranging between $R S S I_{\text {med }}$ and $R S S I_{T X}$ power.

For example, let's consider a system based on a radio receiver with a sensibility of $-95 \mathrm{dBm}$, a TX power transmission of $0 \mathrm{dBm}$ and a medium power of $-60 \mathrm{dBm}$. If the input has a RSSI of $-77.5 \mathrm{dBm}$, the membership degree is $0,0.5$ and 0.5 for each set (HIGH, MEDIUM and LOW). If the input has a RSSI value of $-30 \mathrm{dBm}$, we obtain a membership degree of $0.5,0.5$ and 0 for each fuzzy set.

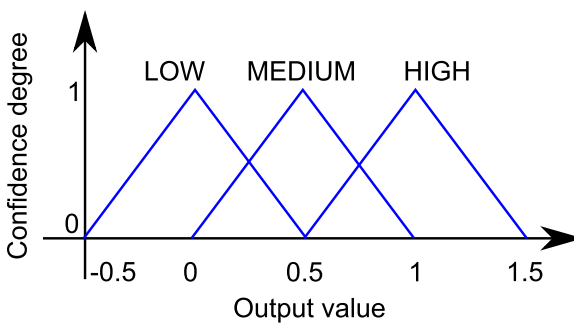

Fig. 11. Sets of the fuzzy output.

\subsubsection{The fuzzy system outputs}

The Fuzzy System offers an output for each and every sector. The output associated to a sector is a $[0,1]$ ranged value that represents the confidence degree (i.e. the truth value obtained as output of the rules of the fuzzy system) in which the tag is actually located in that sector.

As Fig. 11 shows, the LOW output fuzzy set is a triangle with the central point at zero and the corners at -0.5 and 0.5 .

The MEDIUM output is represented by a triangle with the central point at 0.5 and corners at 0 and 1 .

The HIGH output qualifier is also defined by a triangle with the central point at 1 and the corners at 0.5 and 1.5 .

\subsubsection{Inference engine}

The inference engine is the Mandani's rules, with a centroid defuzzification method and a singleton input fuzzificator. The fuzzy engine evaluates the antecedent of every rule by the intersection of the fuzzy inputs, using the minimum function for the AND operator, and the maximum function for the OR operator. In the process of obtaining the output sets as a function of the input sets is called implication. In our system we use the minimum function as the implication process. The implication between the inputs and outputs applies the minimum function.

As mentioned, the rules must be evaluated for every single sector to estimate the confidence degree, taking into account the fuzzy qualifications of RSSI values of either the current sector nodes or the surrounding ones. The rules summed up in Table 1 have been derived from multiple simulations in order to obtain the best trade-off between precision and noise immunity.

It is important to consider that in the application described, it is necessary to know the localization information of the nodes in the Base Station, where it can be accessible for its use. Due to it, obtaining the localization information over the non-anchor nodes, as generally described on typical fully distributed localization algorithms, is not interesting. This is because these devices do not use the above mentioned information. Moreover, obtaining this information could increase battery consumption of the non-anchor nodes. In fact, they are the devices with higher energy constraints.

LIS takes this issue into account. Also, the anchor nodes have more power supply resources than the tags. Consequently, the algorithm has been designed to be executed mainly in the anchor nodes. Furthermore, the radio transceiver of the tag is activated for a short time, which is just 
Table 1

Rules of the inference engine.

\begin{tabular}{|c|c|c|}
\hline $\begin{array}{l}\text { Central RSSI } \\
\text { node }\end{array}$ & RSSI neighbors & Output \\
\hline $\mathrm{HIGH}$ & All MEDIUM & HIGH \\
\hline LOW & All LOW & LOW \\
\hline MEDIUM & All MEDIUM & HIGH \\
\hline MEDIUM & All LOW & LOW \\
\hline $\mathrm{HIGH}$ & All HIGH & MEDIUM \\
\hline MEDIUM & $\begin{array}{l}\text { MEDIUM in current sector } \\
\text { LOW in the rest }\end{array}$ & HIGH \\
\hline MEDIUM & $\begin{array}{l}\text { HIGH in any sector except the current } \\
\text { one } \\
\text { LOW in the rest }\end{array}$ & LOW \\
\hline $\mathrm{HIGH}$ & $\begin{array}{l}\text { High in a neighbor of the current sector } \\
\text { LOW in the rest }\end{array}$ & MEDIUM \\
\hline HIGH & $\begin{array}{l}\text { HIGH in a neighbor, except on the } \\
\text { current sector } \\
\text { LOW in the rest }\end{array}$ & LOW \\
\hline MEDIUM & $\begin{array}{l}\text { MEDIUM in a neighbor of the current } \\
\text { sector } \\
\text { LOW in the rest }\end{array}$ & MEDIUM \\
\hline MEDIUM & $\begin{array}{l}\text { MEDIUM in a neighbor, except on the } \\
\text { current sector } \\
\text { LOW in the rest }\end{array}$ & LOW \\
\hline
\end{tabular}

enough to broadcast the beacon. In the remaining period of time, the tag will be in an idle state and its radio transceiver would be off.

LIS also reduces the power consumption in the anchor nodes. It implements a ubiquitous and distributed algorithm that spreads the localization processing amongst the nodes surrounding the tag. In a centralized-only algorithm, all the information received by the anchor nodes must be delivered to the Base Station. By contrast, the proposed algorithm saves the power energy because only significant information is delivered.

Additional savings can be managed by clustering the networks, and using the cluster heads as the Base Stations, i.e. receiving the processing partial estimations from its cluster nodes.

\subsubsection{Centralized processing stage}

The Base Station collects the partial solutions from the anchor nodes, and processes them cyclically as follows:

C1: The Base Station waits for receiving the first partial solution.

C2: On arrival, the partial solution is saved and a timer starts running.

C3: While the timer is running, the next partial solutions are saved in a table as they were received.

C4: When the timer expires, the system will compute the final position as the centroid of all these partial solutions (triangle sectors). The centroid computation of a finite set of points $\overrightarrow{P_{1}}, \overrightarrow{P_{2}}, \ldots \overrightarrow{P_{N}}$ can be simplified as:

$\overrightarrow{\text { Position }}=\frac{\sum_{i=1}^{N} \overrightarrow{P_{i}}}{N}$
With this algorithm, the Base Station needs to always be active, while waiting to receive any message from the nodes. It determines whether the Base Station is going to consume more than the other nodes. This is not critical because the Base Station acts as a gateway between the sensor network and the external network. Thus, it needs to be placed in an area without power restrictions.

The previous algorithm can be easily extended to locating multiple tags, by simply associating a tag identifier to the transmitted beacons. The final estimated position is time stamped and saved in the Base Station to make it accessible throughout the Internet.

\section{Experimental results}

The localization system was designed to be developed in a Wireless Sensor Network, called ICARO, intended to test an environmental monitorization algorithm. ICARO was placed in the Doñana Biological Reserve.

The Doñana Biological Reserve is a part of the Doñana natural park, a park and wildlife refuge in southwestern Spain. It is located in Andalusia, in the provinces of Huelva, Sevilla and Cadiz, and covers $543 \mathrm{~km}^{2}$, of which $135 \mathrm{~km}^{2}$ are a protected area. The park is an area of marshes, shallow streams, and sand dunes. Doñana Biological Station (DBS) is a Research Institute of the Spanish Council for Scientific Research (CSIC). Some of its main goals are conservation and improving the quality at research in the Doñana Biological Reserve (DBR), which was declared humanity patrimony by UNESCO in 1994 and considered as one of the most important natural protected landscapes in the world. In fact, this year, DBR was included inside the great scientific infrastructures of European Union. In addition, in April 2006, the Spanish Interministerial Comission of Science and Technology (CICYT) from the Spanish Science and Education Department recognized the Doñana Scientific Reserve as a Singular Scientific and Technological Infrastructure (ICTS).

ICARO consists of ten wireless sensors deployed between the zone of "El Ojillo" and "El Zacallon", working on $2.4 \mathrm{GHz}$ ISM band width IEE 802.15.4 Protocol. Fig. 12 shows a map with the current deployment of the system.

This network is based on two kinds of devices given below:

Base station: This is the device that acts as a gateway between the remote measurement sensor and the communication infrastructure of Doñana. It permits the collection of information and allows centralized processing and data fusion. This device is shown in Fig. 13. This system is based on a wireless sensor attached to an industrial PC.

Remote measurement Sensors: These are the devices that permit the acquisition of environmental information. They are powered by solar panels. These devices permit to execute distributed and collaborative algorithms. These devices permit the utilization of data fusion and aggregation for reducing the usage of the bandwidth. These devices are shown in Fig. 14. 


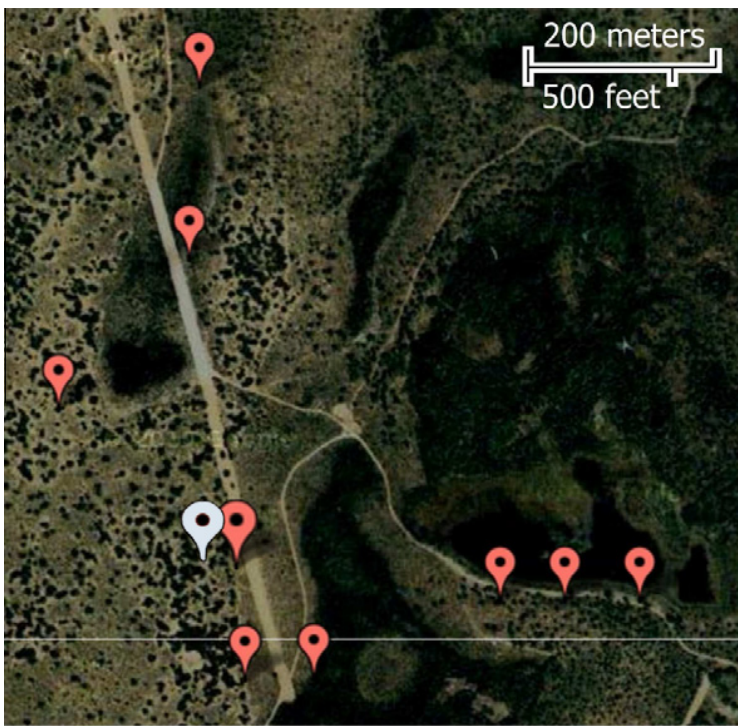

Base Station $\odot$ Anchor node

Fig. 12. Deployment of the project ICARO.

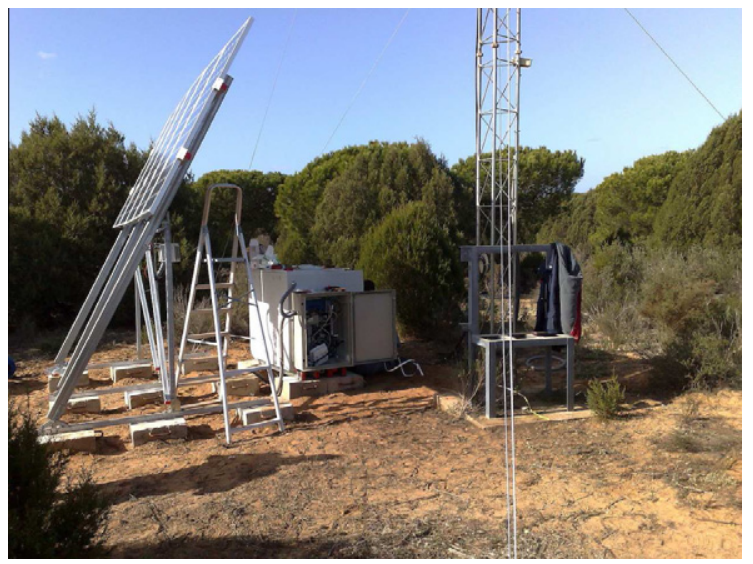

Fig. 13. Base station of the project ICARO.

The current goal consists of the use of the ICARO infrastructure as the anchor nodes, developing a low weight and low cost mobile device, with high autonomy as a non-anchor node for tracking animals in the Doñana Natural Park, such as the device shown on Fig. 15. To do this, the Base station can be used to execute the centralized algorithm of the localization, while the remote measurement sensor executes the distributed algorithm as the anchor nodes.

\subsection{Relationship between RSSI and distance}

Fig. 16 shows the experiments to evaluate the relationship between RSSI and the distance in different situations: free-space without obstacles and long distance with obstacles.

These graphics are obtained by increasing the distance between a node acting as an emitter and another node

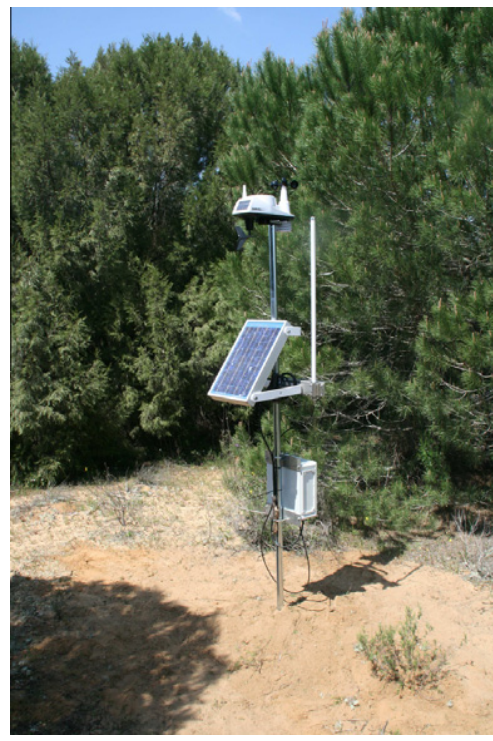

Fig. 14. Remote measurement sensor of the project ICARO.

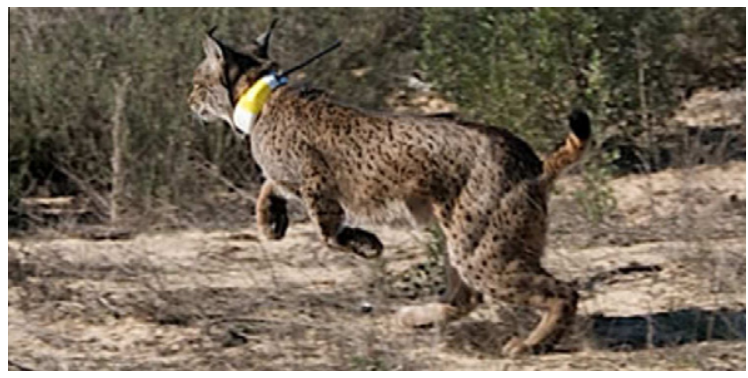

Fig. 15. Example of non-anchor node for tracking animals.

acting as a receiver, waiting for the time for stabilization of the system and acquiring a minimum of 50 values per point represented on the graphic. These data shows the medium value, trying to reduce the effect of the noise in order to obtain the relationship between distance (in [5-50 $\mathrm{m}$ ] range) and RSSI (in $\mathrm{dBm}$ ).

The measured standard deviation has been approximately about $3 \mathrm{dBm}$. It was detected that the measurement fluctuations are higher in short distances that in far off distances.

The "free space" results are measured in the park, in a dessert zone, without trees or grass in the area that could produce disturbances. The results with "obstacles" are the measurements in a corridor between the trees with around $15 \mathrm{~m}$ of height, where there are small plants and grass.

The results with obstacles do not match with any valid mathematical model that could directly permit to obtain the distance, using only the RSSI.

Obtaining models with the relationship between RSSI and distance is currently an important area of research. In any case, it is possible to obtain this model with a much studied environment. Fig. 17 shows an example developed by Texas Instruments [58], where the RSSI value was 


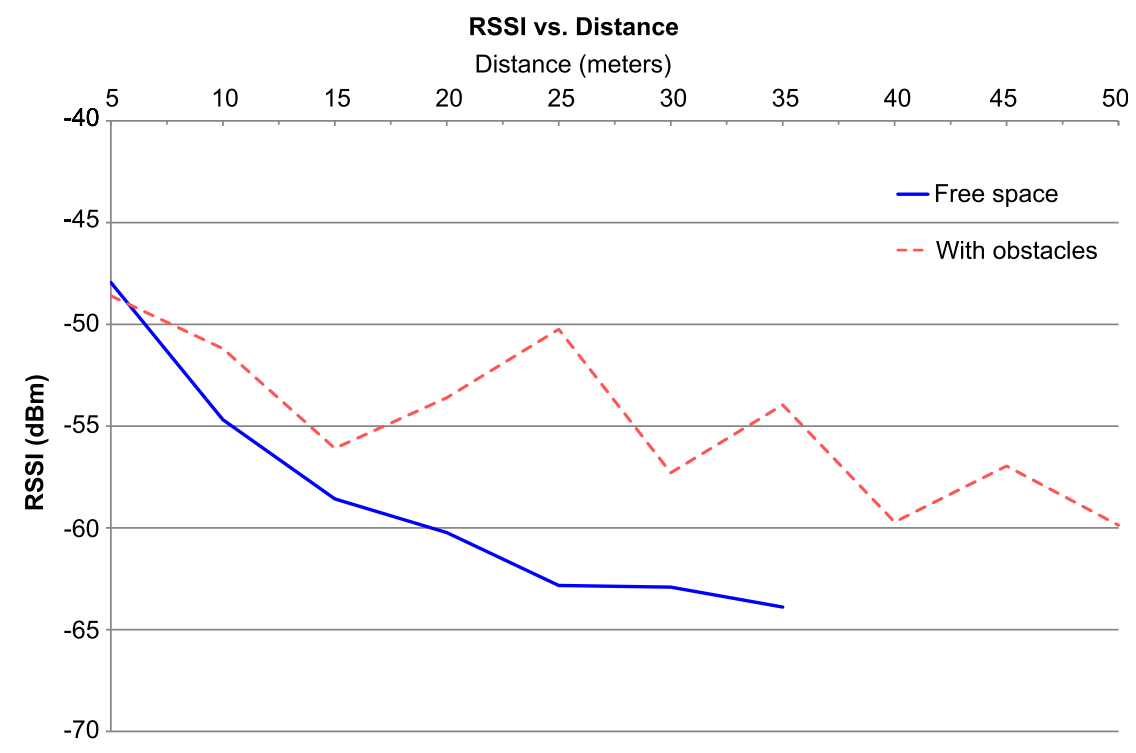

Fig. 16. RSSI vs. distance.

measured and obtained when a beacon sent from a node was received by another node. This figure shows the evaluation of the received RSSI at different elevations from the floor. In the application note [58], Texas Instruments evaluates these information using two mathematical models: Friis and ground model.

The radio transceiver used is the CC2420, which is frequently used in WSN devices. In fact, this is the radio transceiver used in the current implementation.

However, the main problem is to obtain the inverse of this relationship. In fact different distances produced the same RSSI value. Thus, even having a correct model of the environment and ideal situations, it is not possible to obtain the distance using only the RSSI information. In this case, it is necessary to use additional information, such as using multiple receivers or assume simplifications and the errors that can be produced. The proposal algorithm uses the connectivity information about the anchor nodes as additional information to obtain the position. In addition, the fuzzy system improves the behavior against the noise.

\subsection{Simulations}

The accuracy of LIS versus the classic CL algorithm [42] was compared using different simulations. The tested network was made up of a non-anchor node and 25 anchor devices with a radio range of $200 \mathrm{~m}$ and with separated anchor nodes also with a radio range of about $200 \mathrm{~m}$ (Fig. 18). The anisotropic radiation pattern was assumed.

The election of centroid as the reference algorithm to compare with is based on the fact that many authors use it in their research studies. Thus, it is possible to obtain a conclusion on the accuracy of LIS not only with the centroid, but also with all the other localization techniques that are compared with it in the literature.
This section describes the simulator and the results obtained with it. The use of a simulator for obtaining the accuracy of a localization algorithm is a common tool. It is difficult to compare the localization algorithms in real scenarios, because of the changes of the environment. Thus, these experiments are generally not replicable, even by the author (different days would offer different results). Obtaining unified comparison criteria for the localization algorithm and the necessary parameters to consider in the real experiments is currently an important area of research, which is far from over. However, simulation results are easily reproducible and permit the comparison of the localization algorithms amongst themselves.

\subsubsection{Description of the simulator}

The simulator has been developed in C++ (Fig. 19). It allows the selection of the radio range, radiation pattern, Gaussian noise, sensibility, network deployment and anchor location. All the parameters, in the tests have been selected to model the TelosB devices. The results of the simulations are presented in the next subsection.

\subsubsection{Error vs. position}

The following experiments include a moving tag into the network. The noise has been neglected and the error is expressed in meters. As Figs. 20-22 show, the maximum and average errors of LIS algorithm are considerably smaller than the ones estimated with the (CL) Centroid classic algorithm.

Figs. 20 and 21 are graphical representations of the errors, which represent the real position and the estimated position of the algorithms in a few analyses. The results are normalized with the radio coverage of the non-anchor nodes that are $200 \mathrm{~m}$ in these tests.

Fig. 22 represents the position error, measured as the absolute value of the Euclidean distance between the real and the estimated position. The results are normalized 


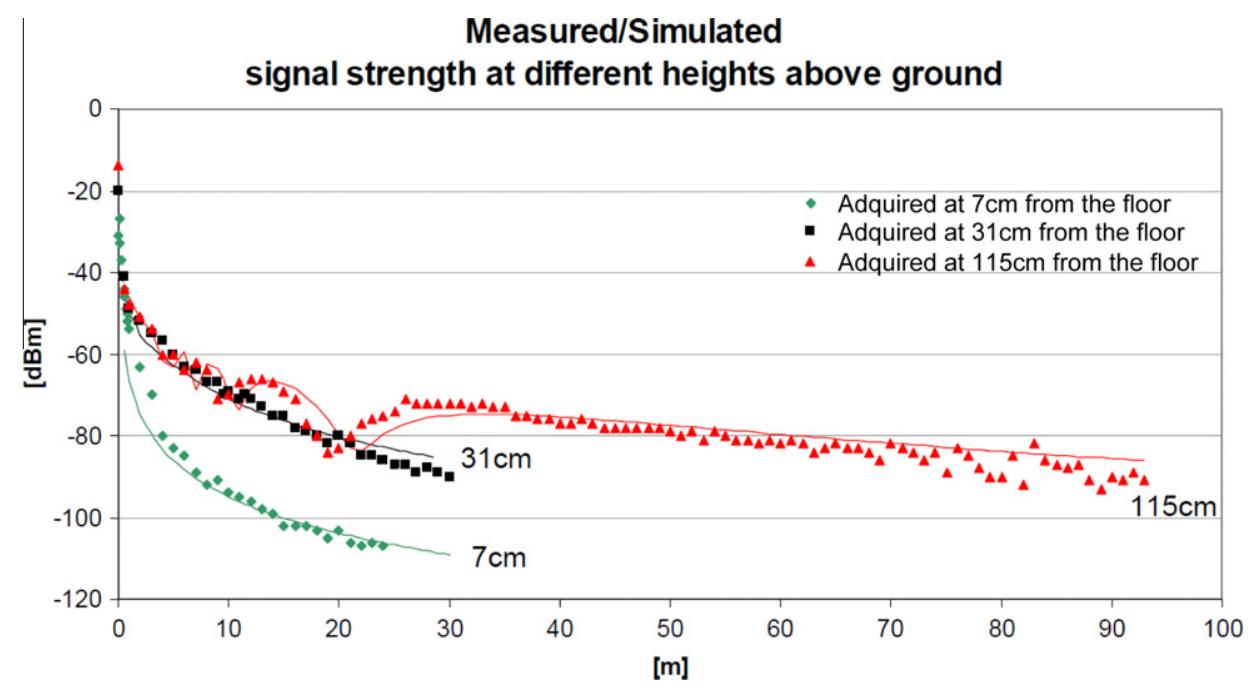

Fig. 17. Evaluation of the RSSI in open field. Results obtained by Texas Instrument [58].

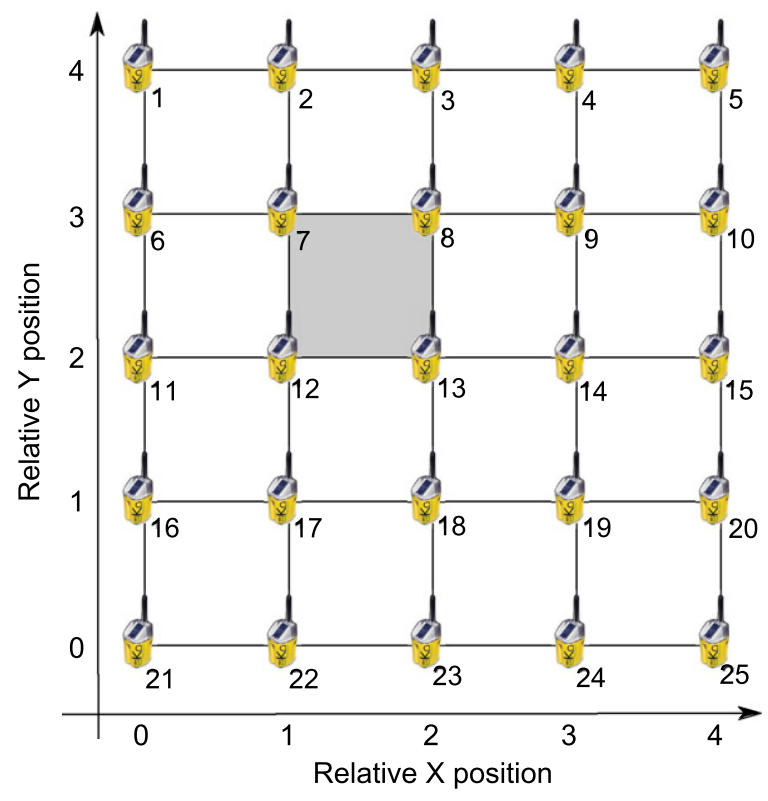

Fig. 18. Simulated network.

with the non-anchor node radio range. They are obtained by moving the non-anchor node into the square made up by the anchor nodes 7, 8, 9 and 12, in a step of $2 \mathrm{~m}$. The $X$ axis represents the relative position of the tag into the square, assuming that node 8 is the coordinates origin. These values are normalized with the distance between then nodes (200 meters). The last relative point simulated is $[0.5,0.5]$ because the other three parts of the square offer symmetrical results than obtained result.

The obtained result shows that in all the simulated points, LIS presents an error equal or minor than CL algorithm. The error obtained is similar than the error that can be obtained with the other range-free techniques. However, the LIS performance is better in noisy conditions.

\subsubsection{Error vs. coverage}

Fig. 23 shows the influence of the radio range. As the radio range increases, the number of non-anchor nodes that receive a beacon also increases, and the error decreases. In this scenario the noise has not been considered. The radio range of the non-anchor node simulated is in the [100-350 m] range.

Results are the relative error to the distance between the anchor nodes that are separated 200 meters. There are six different points, which are simulated corresponding to the points 1 to 6 represented in the figure, separated by $50 \mathrm{~m}$. These points are into the square of the nodes $7,8,12$ and 13 of the network. The rest of the points can be obtained by dividing the square in step of $50 \mathrm{~m}$, which offers identical results than those of the simulated. This is because of the symmetry.

In all of the cases, LIS gets smaller or equal errors than the centroid algorithm. Similar results are obtained by fixing the coverage area of the non-anchor nodes and reducing the distance between the anchor devices from the simulated $200 \mathrm{~m}$. These results are similar to the evaluation of the error versus the node density, which was proposed by the other authors.

As it can be seen in points where there is symmetry between the position of the tags and the anchor nodes (points 1,3 and 6 ), the error is always 0 for all the simulated coverage. This is a typical behavior of the centroid, which has low errors in these symmetrical points, but has very bad behavior outside these points. LIS presents a good behavior in all of the simulated points.

With the two algorithms, better accuracy is obtained by increasing the number of anchor nodes that receives the beacon, but with the proposed method, the system tends to lower the errors earlier.

\subsubsection{Error vs. noise}

In this experiment the error obtained in a fixed position of the non-anchor nodes when changing the variance of the Gaussian noise was analyzed. All the experiments have used a coverage area of $200 \mathrm{~m}$ for the non-anchor node and 


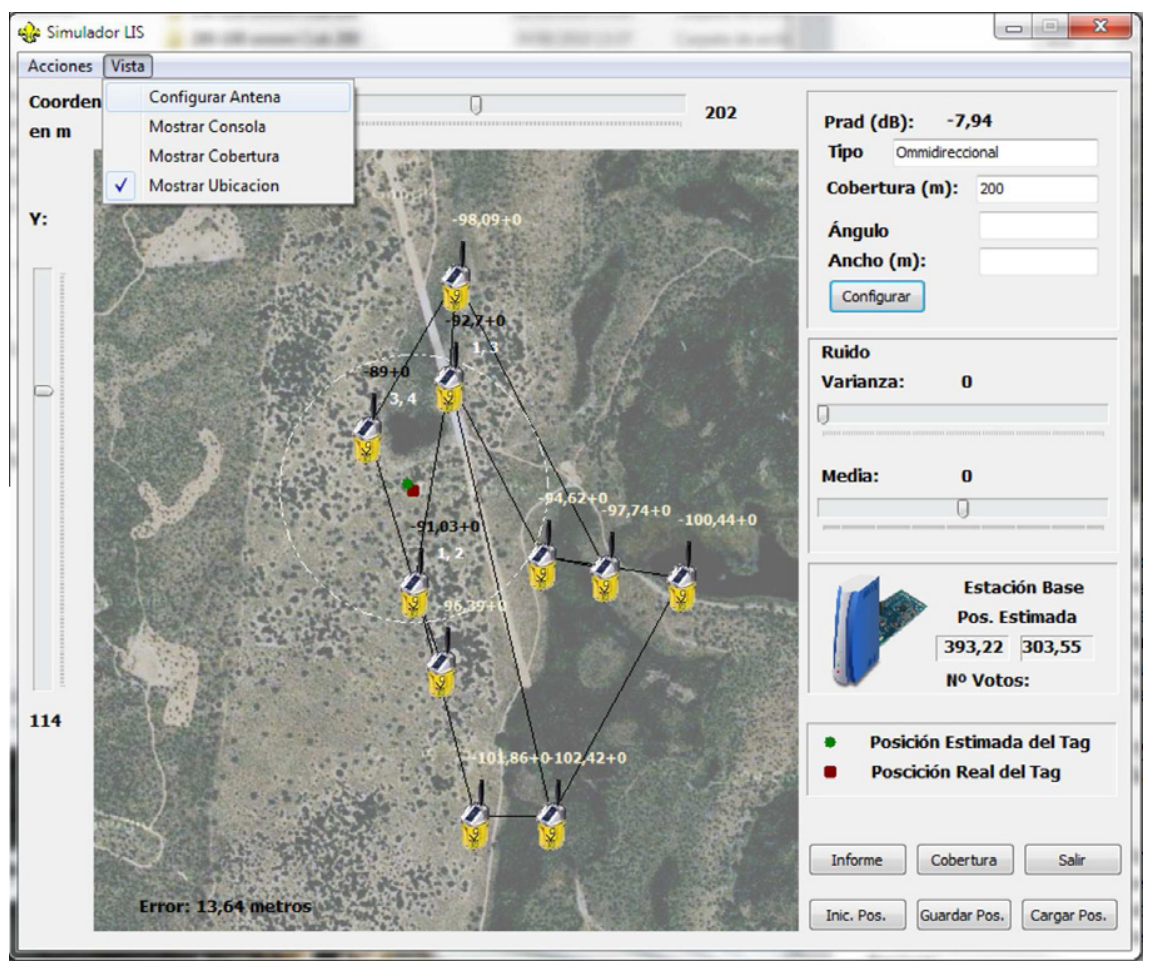

Fig. 19. LIS simulator.

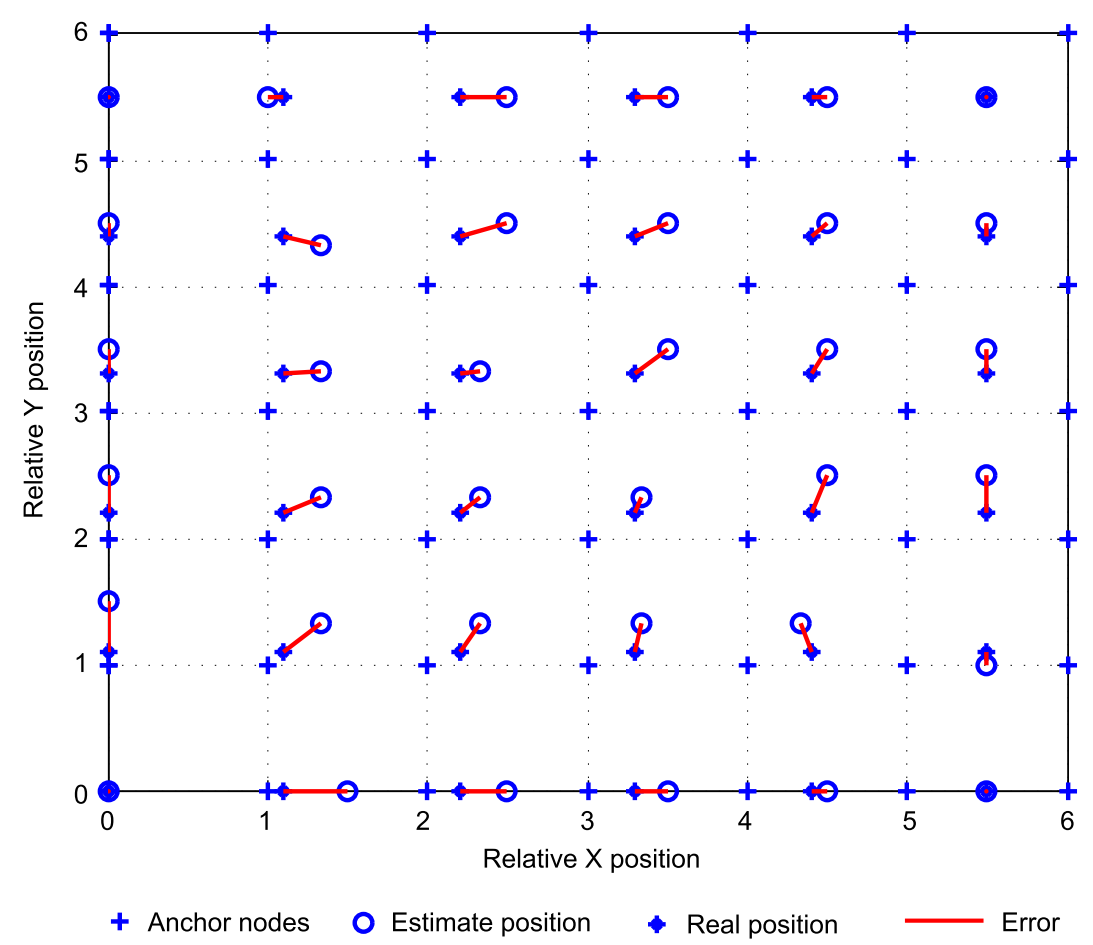

Fig. 20. Position error of centroid algorithm. 


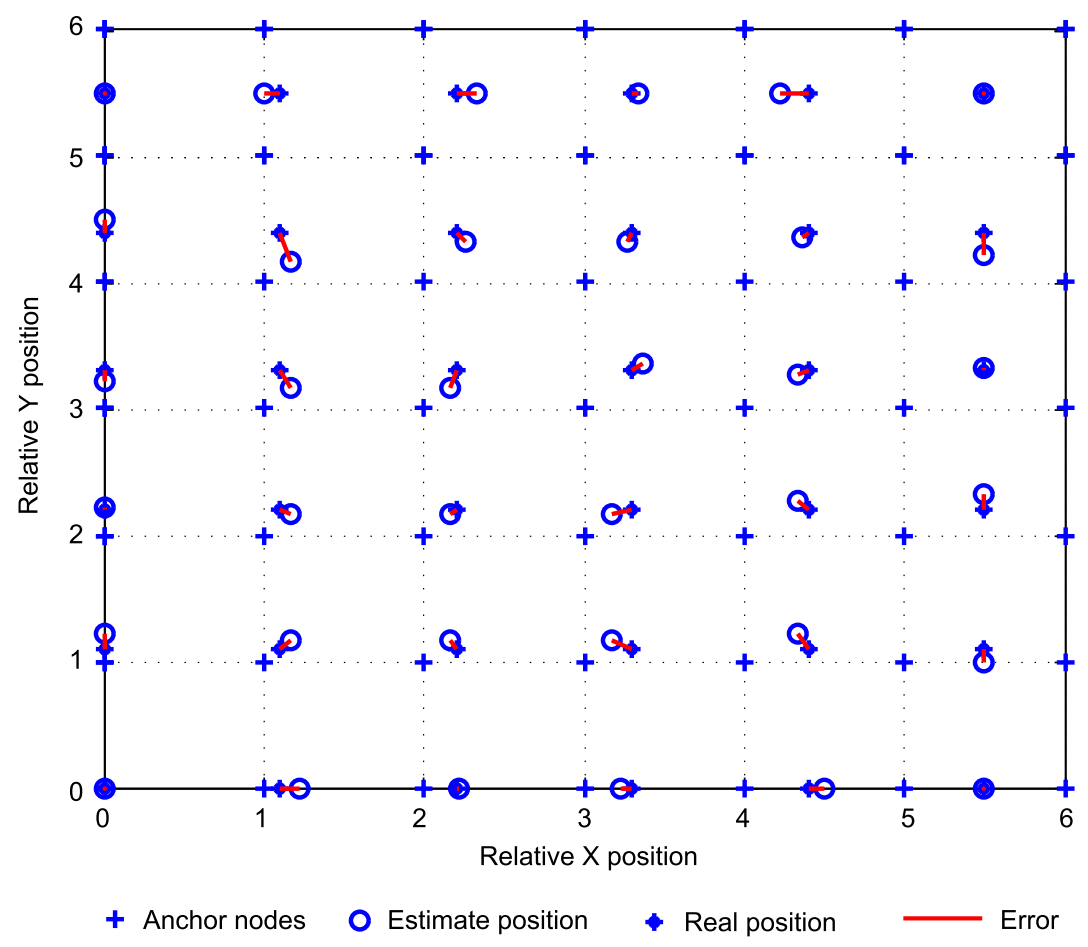

Fig. 21. Position error of LIS algorithm.

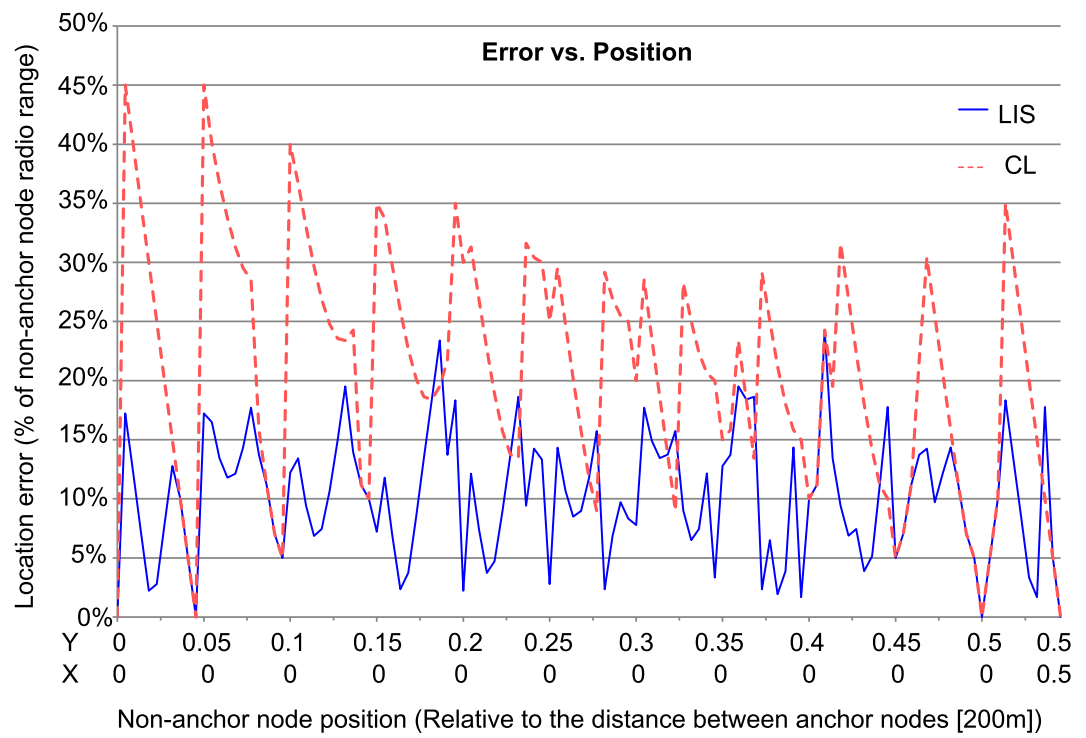

Fig. 22. Localization error vs. the position of the non-anchor node.

have been done in the same six points comments as the previous section. To obtain these results 1000 analyses were made at every point and the absolute Root Mean Square (RMS) localization error was measured. The represented results are normalized with the non-anchor node radio range.

The error vs. variance (Fig. 24) represents how the noise affects the accuracy of the system. The centroids have a good behavior on points of symmetry with respect to the anchor nodes (such as point 1, 3 and 6), but have a worse behavior on the resting point. Moreover, LIS offers similar responses in any localization, thereby obtaining good results on the non-symmetrical points.

The number of errors (Fig. 25) represents the number of absolute errors bigger than $100 \mathrm{~m}$ ( $1 / 2$ of the coverage area) obtained in the localization. If the tag is situated in the center of the square, this error represents the number of results that situate the device out of the square. This is 


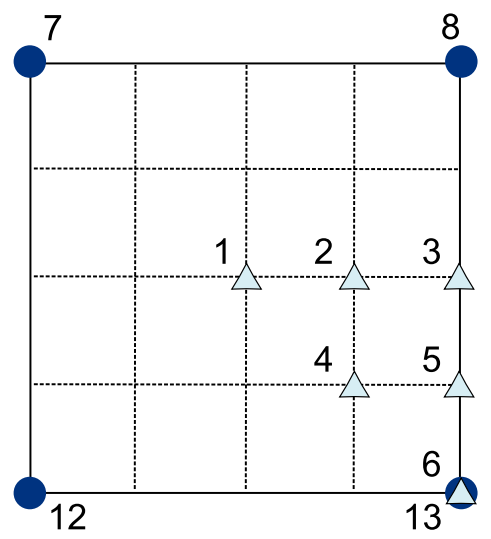

- Anchor nodes

\section{$\triangle$ Non-anchor node Positions}
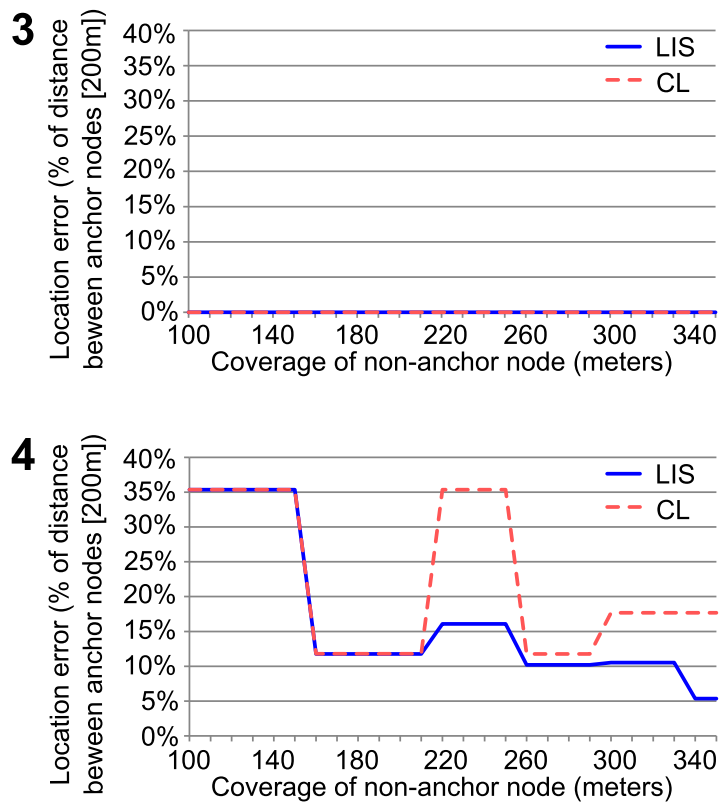
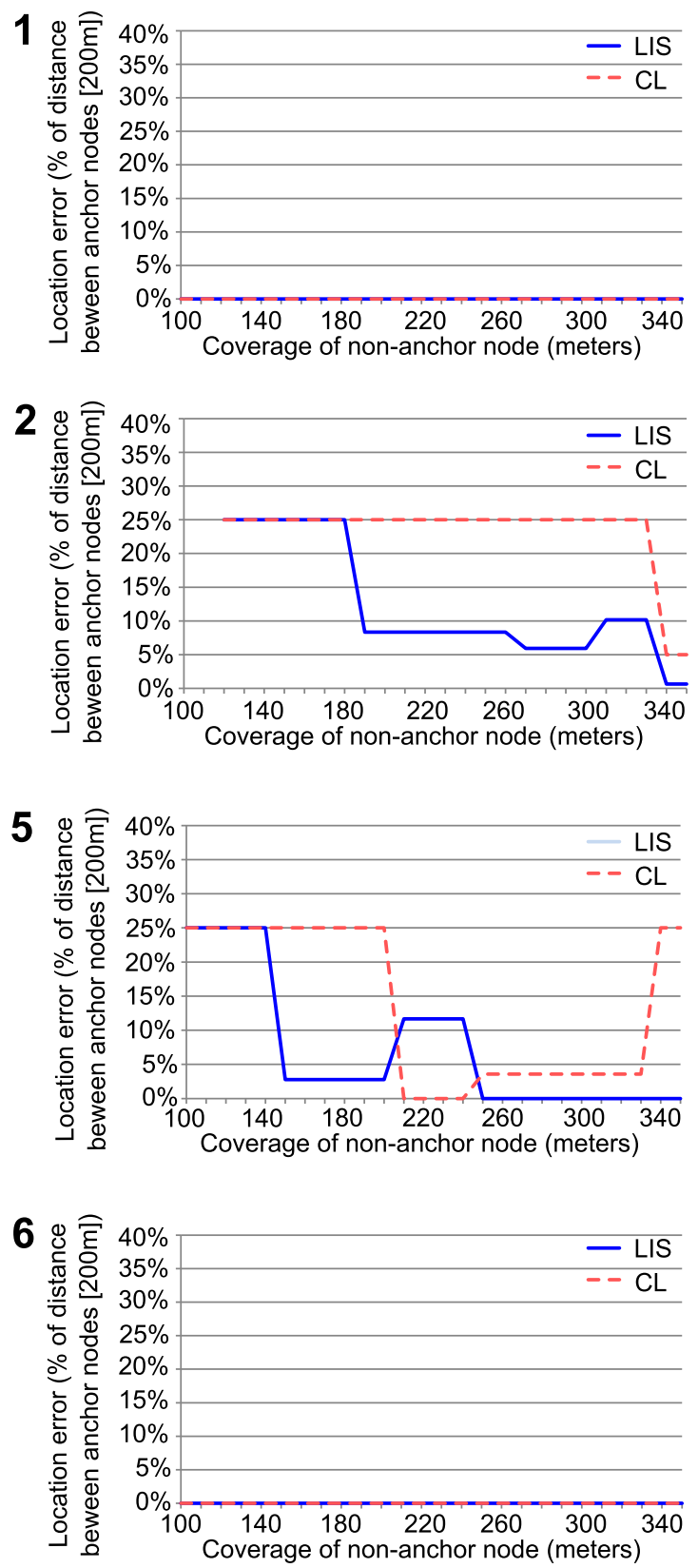

Fig. 23. Localization error vs. the coverage radio area of non-anchor node.

in relationship with the metrics and is called tile accuracy. The tile accuracy value, intended for this point can be obtained 100 to the represented values, expressed in percentages. These metrics are defined for the range-free techniques as the systems are generally applied for determining if a tag is into an area, more than for offering a punctual position.

As it can be seen, LIS always has a less number of errors bigger than $100 \mathrm{~m}$ than the classic CL algorithm, especially in the non-symmetrical points.

It is important to note that the LIS algorithm estimates the position of the tag without applying any filter to the obtained data. Obtaining a method to filter the results, and increasing the accuracy, as described in the next section, is under investigation by the authors.

\subsection{Real experiments}

All the real experiments have been done over the ICARO infrastructure. Fig. 26 represents a mesh of the current network and the connectivity between the devices.

The experiments have been done locating the non-anchor node into the sector made by the anchor nodes 1,2 and 3 . In these experiments a node has been used as a 


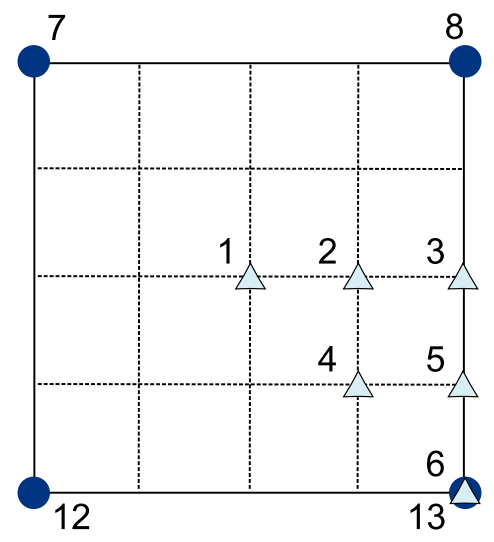

Anchor nodes

Non-anchor node Positions
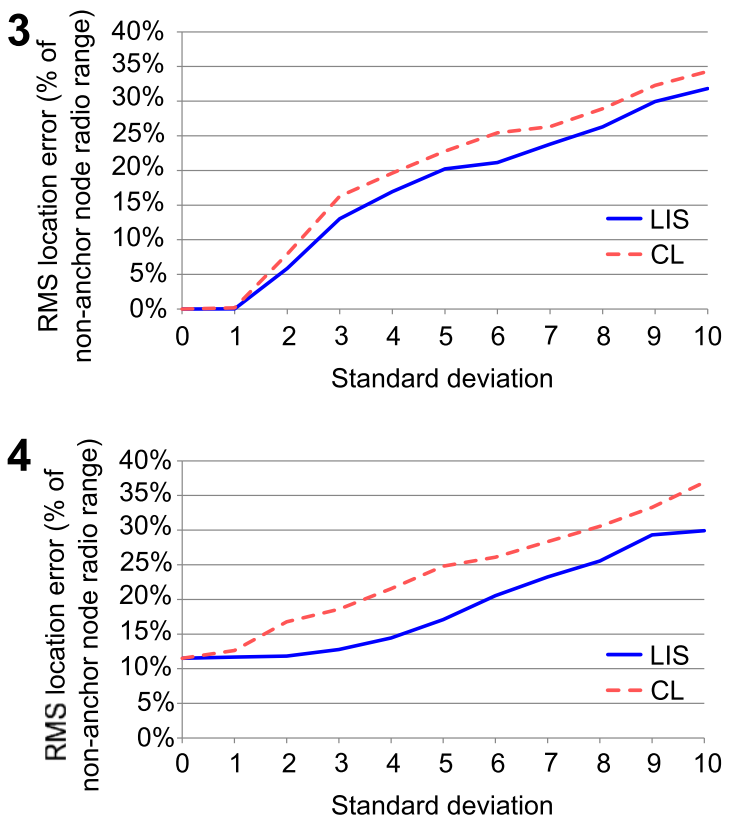
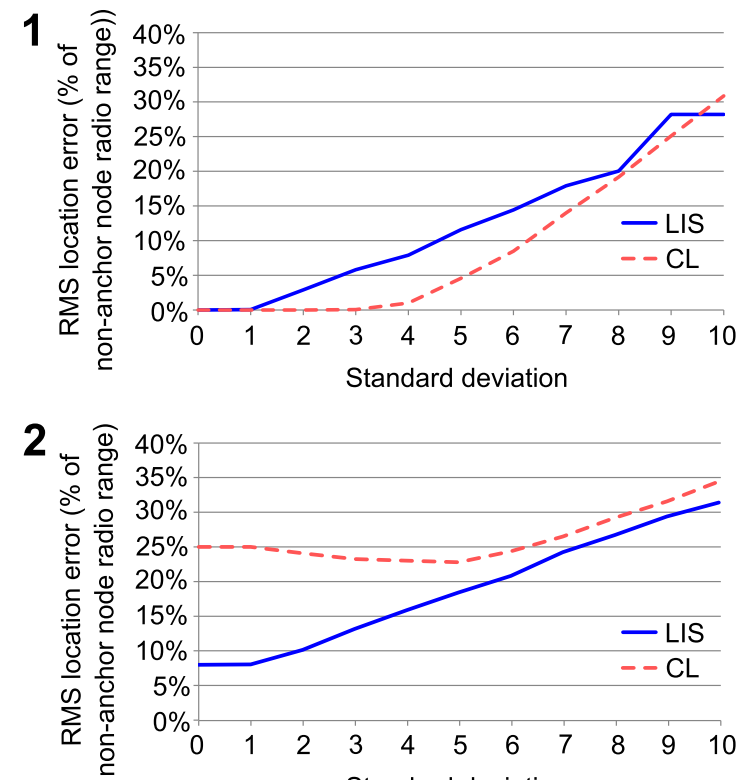

Standard deviation

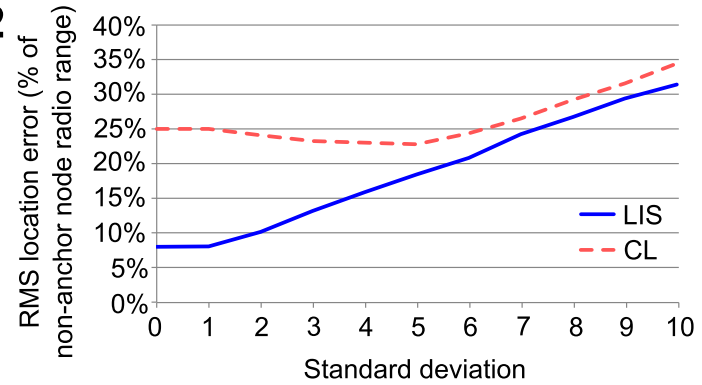

5

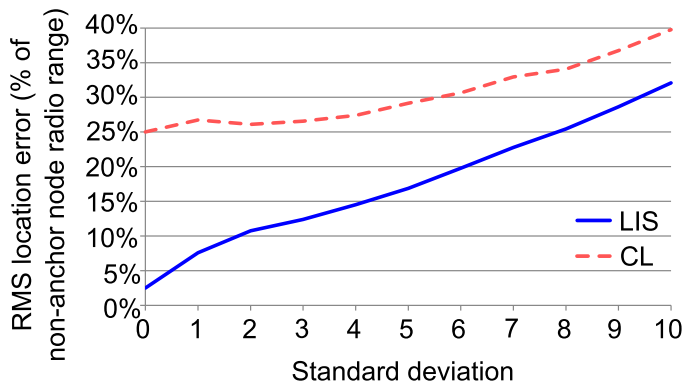

6

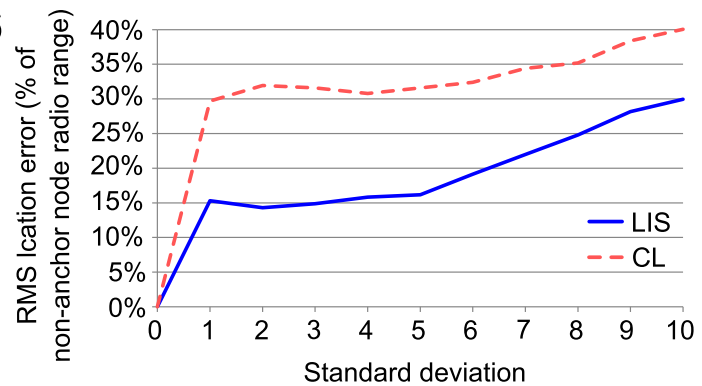

Fig. 24. Localization error vs. the noise.

tag with the same characteristic than the anchor nodes: a CC2420 radio transceiver with an omnidirectional $8 \mathrm{dBi}$ antenna. This tag offered about $300 \mathrm{~m}$ of radio range in the current experiments.

For these experiments it had been necessary to calibrate the devices. The calibration consisted of adjusting the RSSI values. The procedure to calibrate the RSSI measurement consisted of the following steps:

1. Measurement of the RSSI level at the minimum distance - this is the tag is next to the anchor node $(0 \mathrm{~m})$.

2. Measurement of the RSSI at the maximum radio coverage $(300 \mathrm{~m})$.
3. Measurement of the RSSI at the medium radio coverage distance $(150 \mathrm{~m})$.

The measured position RMS error locating the tag at the center of the area is low (around a 15\% of the non-anchor node radio range). Moving the non-anchor node away from this position to the center of one of the sides of the triangle makes the RMS error to grow up to $35 \%$ of the non-anchor node radio range.

It is important to consider that the actual network is not very adequate to test the localization algorithm, because the connectivity between nodes is low. Initially, this network has been developed for the ambient monitoring in the 


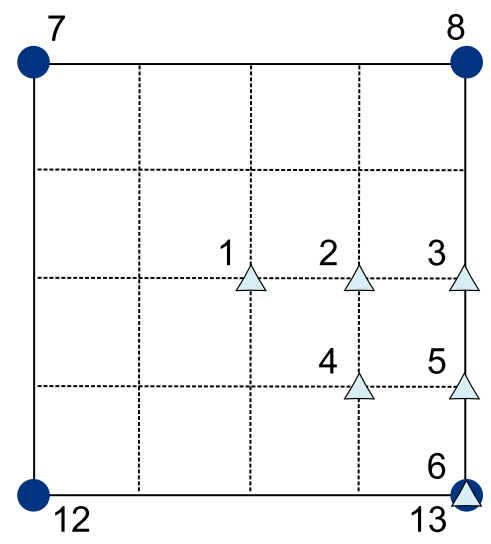

- Anchor nodes

\section{$\triangle$ Non-anchor node Positions}
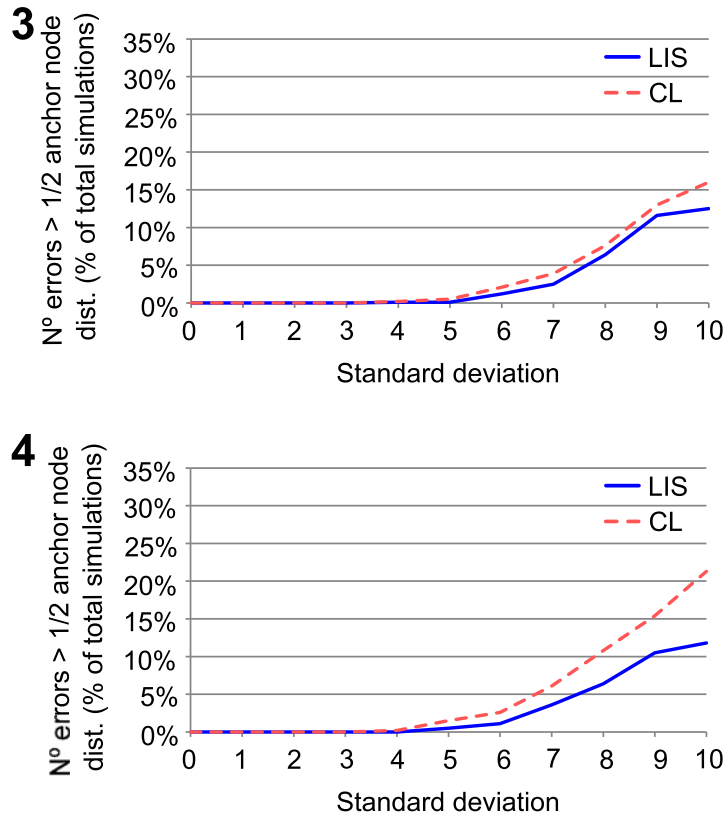
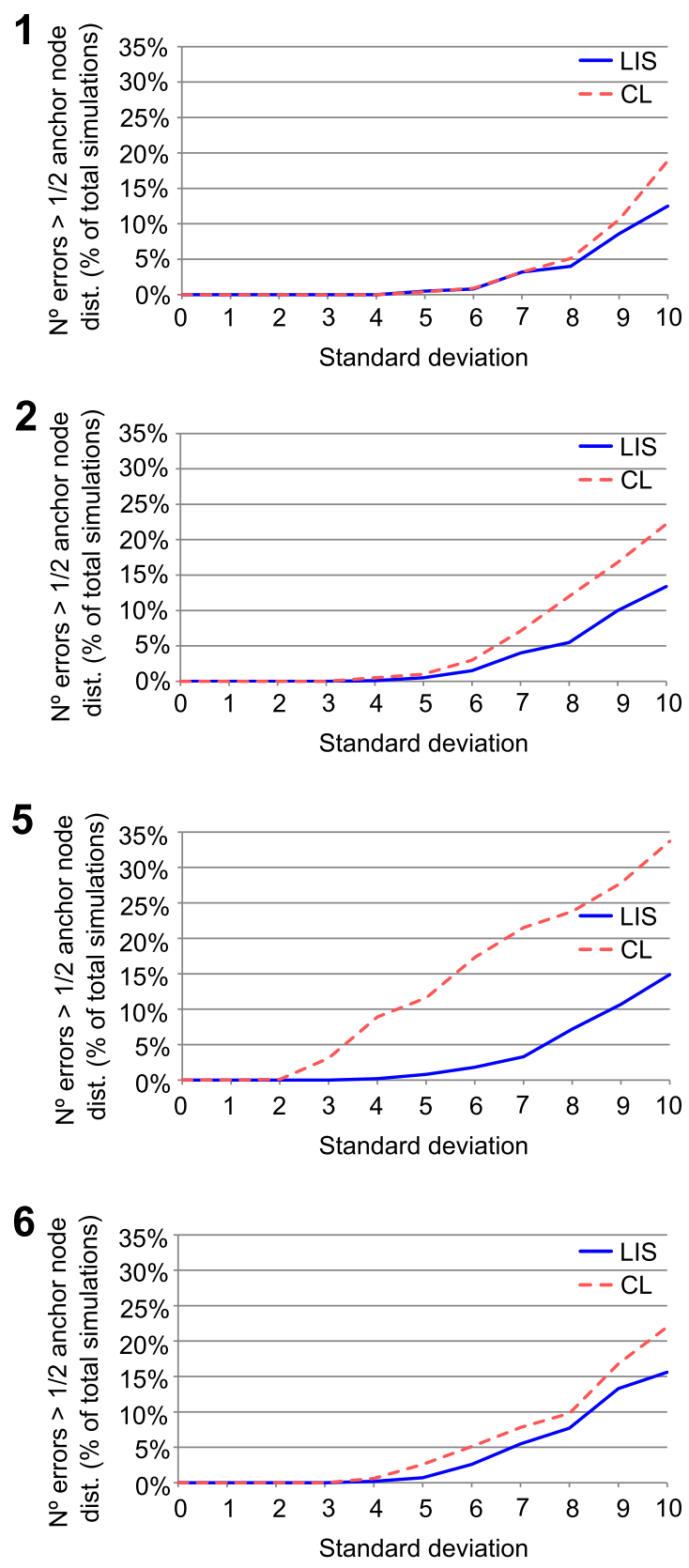

Fig. 25. Number of error bigger than $100 \mathrm{~m}$.

marsh zones of the natural park of Doñana. Currently, the authors are studying the increase in the number of anchor nodes, to obtain a reliable benchmark for localization algorithms.

\section{Conclusions and future work}

LIS is a new fuzzy algorithm for localization designed to reduce power consumption, especially but not limited to, the tag nodes where the power constraints are higher. LIS filters the useless information after being processed in the anchor nodes. It also implements a hibernation mechanism. All these mechanisms increase the battery autonomy.

LIS has been tested by simulations and in a real scenario. The obtained results showed that the proposed method obtains less localization errors than the $\mathrm{CL}$ algorithm without higher computation requirements or an extensive use of radio.

The localization system LIS is being applied to locating and tracking of wild animals in natural parks.

Currently, the aim is to increase the accuracy of the system versus the noise, acting along the following lines: 


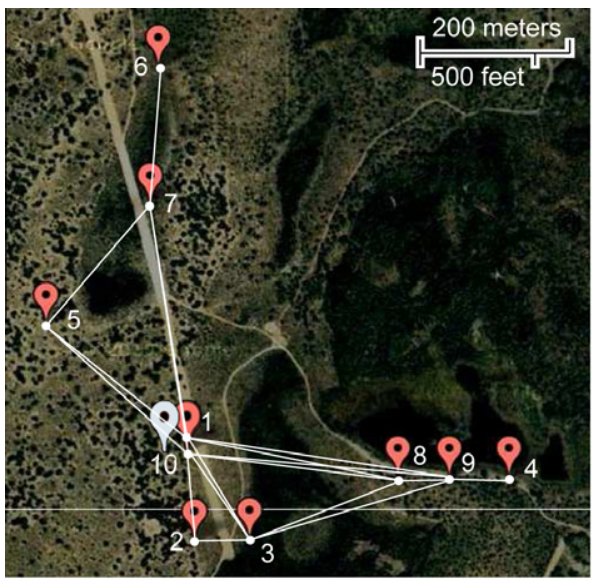

(a)

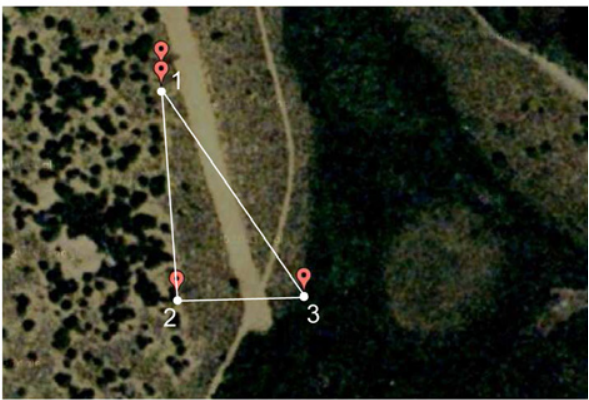

(b)

Fig. 26. (a) Mesh representation of the links in the ICARO network, and (b) detailed area.

- Using a filter to reduce the variability of the measurement: The current study attempts to work on the evaluation of the advantages of using filters to RSSI measures for increasing the accuracy.

- Use additional information of RSSI between nodes: Current radio devices can provide RSSI information for every message, which is more than what the radio can intercept. For example, the radio message sender between the anchor nodes can be used in the localization to model the environment without adding traffic between the devices.

- Use Temporal information: The current proposed method only uses the information obtained in a predetermined instant; it does not have past memory. The current study attempts to work on a modification to the current algorithm, which improves its performance using this information.

\section{Acknowledgments}

This research has been supported by the "Consejería de Innovación, Ciencia y Empresa", "Junta de Andalucía”, Spain, through the excellence project ARTICA (reference number: P07-TIC-02476) and by the "Cátedra de Telefónica, Inteligencia en la Red”, Seville, Spain, through the Project ICARO.

The authors thank the Biological Station of the natural park of "Doñana" and the researchers of its Biological Station Centre, for their collaboration and support.

\section{References}

[1] J.A. Gutierrez, M. Naeve, E. Callaway, M. Bourgeois, V. Mitter, B. Heile, IEEE 802.15.4: a developing standard for low-power low-cost wireless personal area networks, IEEE Network 15 (2001) 12-19.

[2] P. Baronti, P. Pillai, V.W.C. Chook, S. Chessa, A. Gotta, Y.F. Hu, Wireless sensor networks: a survey on the state of the art and the 802.15.4 and ZigBee standards, Comput. Commun. 30 (2007) 1655-1695.
[3] Z. Hu, B. Li, On the fundamental capacity and lifetime limits of energy-constrained wireless sensor networks, in: Proc. IEEE Real Time Embedded Technol. Symp., vol. 10, 2004, pp. 2-9.

[4] J. Polastre, R. Szewczyk, D. Culler, Telos: enabling ultra-low power wireless research, in: Fourth International Symposium on Information Processing in Sensor Networks, Proc. IPSN, 2005, pp. 364-369.

[5] W. Ye, J. Heidemann, D. Estrin, An energy-efficient MAC protocol for wireless sensor networks, Proc. IEEE INFOCOM 3 (2002) 1567-1576.

[6] V. Raghunathan, C. Schurgers, S. Park, M.B. Srivastava, Energy-aware wireless microsensor networks, IEEE Signal Process. Mag. 19 (2002) 40-50.

[7] B. Chen, K. Jamieson, H. Balakrishnan, R. Morris, Span: an energyefficient coordination algorithm for topology maintenance in ad hoc wireless networks, in: Proc. Annu. Int. Conf. Mobile Comput. Networking, 2001, pp. 85-96.

[8] G. Sudha Anil Kumar, G. Manimaran, Z. Wang, Energy-aware scheduling with probabilistic deadline constraints in wireless networks, Ad Hoc Networks 7 (2009) 1400-1413.

[9] Y. Wu, S. Fahmy, N.B. Shroff, Sleep/wake scheduling for multi-hop sensor networks: non-convexity and approximation algorithm, Ad Hoc Networks 8 (2010) 681-693.

[10] I.F. Akyildiz, W. Su, Y. Sankarasubramaniam, E. Cayirci, Wireless sensor networks: a survey, Comput. Networks 38 (2002) 393422.

[11] J. Yick, B. Mukherjee, D. Ghosal, Wireless sensor network survey, Comput. Networks 52 (2008) 2292-2330.

[12] J.A. López Riquelme, F. Soto, J. Suardíaz, P. Sánchez, A. Iborra, J.A. Vera, Wireless sensor networks for precision horticulture in Southern Spain, Comput. Electron. Agric. 68 (2009) 25-35.

[13] W. Chung, Y. Lee, S. Jung, A wireless sensor network compatible wearable U-healthcare monitoring system using integrated ECG accelerometer and SpO2, in: Proc. Annu. Int. Conf. IEEE Eng. Med. Biol. Soc., EMBC - Pers. Healthc. through Technol., 2008, pp. 15291532.

[14] T. He, S. Krishnamurthy, J.A. Stankovic, T. Abdelzaher, L. Luo, R. Stoleru, et al., Energy-efficient surveillance system using wireless sensor networks, in: MobiSys Second Int. Conf. Mobile Syst. Appl. Serv., 2004, pp. 270-283.

[15] A. Boukerche, H.A.B. F Oliveira, E.F. Nakamura, A.A.F. Loureiro, Secure localization algorithms for wireless sensor networks, IEEE Commun. Mag. 46 (2008) 96-101.

[16] T. Antoine-Santoni, J. Santucci, E. de Gentili, X. Silvani, F. Morandini, Performance of a protected wireless sensor network in a fire, Anal. Fire Spread Data Trans., Sensors 9 (2009) 5878-5893.

[17] M. Tubaishat, Z. Peng, Q. Qi, S. Yi, Wireless sensor networks in intelligent transportation systems, Wireless Commun. Mobile Comput. 9 (2009) 287-302.

[18] W. Ren, A rapid acquisition algorithm of WSN-aided GPS location, in Proc. Int. Symp. Intell. Inf. Technol. Secur. Informatics, IITSI., 2009, pp. $42-46$. 
[19] E. Elnahrawy, X. Li, R.P. Martin, The limits of localization using signal strength: a comparative study, in: First Annu. IEEE Commun. Soc. Conf. Sensor Ad Hoc Commun. Netw. IEEE SECON., 2004, pp. 406414.

[20] G. Zanca, F. Zorzi, A. Zanella, M. Zorzi, Experimental comparison of RSSI-based localization algorithms for indoor wireless sensor networks, in: REALWSN - Proc. Workshop Real-World Wirel. Sensor Netw., 2008, pp. 1-5.

[21] S.A. Mitilineos, J.N. Goufas, O.E. Segou, S.C.A. Thomopoulos, WAXROOM: an indoor WSN-based localization platform, in: Proc. SPIE Int. Soc. Opt. Eng., vol. 7697, 2010.

[22] F. Reichenbach, J. Blumenthal, D. Timmermann, Comparing the efficiency of localization algorithms with the Power-Error-Product (PEP), in: Proc. Int. Conf. Distrib. Comput. Syst., 2008, pp. 150-155.

[23] E. Cassano, F. Florio, F. De Rango, S. Marano, A performance comparison between ROC-RSSI and trilateration localization techniques for WPAN sensor networks in a real outdoor testbed, Wirel. Telecommun. Symp. WTS. (2009).

[24] A. Awad, T. Frunzke, F. Dressler, Adaptive distance estimation and localization in WSN using RSSI measures, in: Proc. - Euromicro Conf. Digit. Syst. Des. Archit., Methods Tools, DSD., 2007, pp. 471-478.

[25] S. Wu, N. Zhang, Two-step TOA estimation method for UWB based wireless sensor networks, Ruan Jian Xue Bao. 18 (2007) 1164-1172.

[26] S. Xiaoyan, L. Jiandong, H. Pengyu, P. Jiyong, Total least-squares solution of active target localization using TDOA and FDOA measurements in WSN, in: Proc. Int. Conf. Adv. Inf. Netw. Appl. AINA., 2008, pp. 995-999.

[27] D. Niculescu, B. Nath, Ad hoc positioning system (APS) using AOA, Proc. IEEE INFOCOM 3 (2003) 1734-1743.

[28] P. Rong, M.L. Sichitiu, Angle of arrival localization for wireless sensor networks, sensor and ad hoc communications and networks, 2006, in: SECON '06. 2006 3rd Annual IEEE Communications Society on., vol. 1, 2006, pp. 374-382.

[29] S. Wang, K. Shih, C. Chang, Distributed direction-based localization in wireless sensor networks, Comput. Commun. 30 (2007) 14241439.

[30] K. Kucuk, A. Kavak, H. Yigit, C. Ozdemir, A novel localization technique for wireless sensor networks using adaptive antenna arrays, IEEE Radio Wirel. Symp., RWS. (2008) 483-486.

[31] D. Niculescu, B. Nath, Localized positioning in ad hoc networks, Ad Hoc Networks 1 (2003) 247-259.

[32] D. Niculescu, B. Nath, Position and orientation in ad hoc networks, Ad Hoc Networks 2 (2004) 133-151.

[33] K. Kucuk, A. Kavak, Scalable location estimation using smart antennas in wireless sensor networks, Ad Hoc Networks 8 (2010) 889-903.

[34] T. Sathyan, M. Hedley, Cooperative localization - algorithms for initial position estimation, in: ISSNIP - Proc. Int. Conf. Intelligent Sens., Sens. Netw. Inf. Process., 2008, pp. 13-18.

[35] A. Chehri, P. Fortier, P.M. Tardif, UWB-based sensor networks for localization in mining environments, Ad Hoc Networks 7 (2009) 987-1000.

[36] R. Eickhoff, F. Ellinger, R. Mosshammer, R. Weigel, A. Ziroff, M. Huemer, 3D-accuracy improvements for TDoA based wireless local positioning systems, IEEE Globecom Workshops, GLOBECOM (2008).

[37] J. Hwang, T. He, Y. Kim, Secure localization with phantom node detection, Ad Hoc Networks 6 (2008) 1031-1050.

[38] F. Su, W. Ren, H. Jin, Localization algorithm based on difference estimation for wireless sensor networks, in: Proc. Int. Conf. Commun. Softw. Netw., ICCSN., 2009, pp. 499-503.

[39] H. Miura, K. Hirano, N. Matsuda, H. Taki, N. Abe, S. Hori, Indoor localization for mobile node based on RSSI, Lect. Notes Comput. Sci. 4694 LNAI (2007) 1065-1072.

[40] S. Tian, X. Zhang, P. Liu, P. Sun, X. Wang, A RSSI-based DV-hop algorithm for wireless sensor networks, Int. Conf. Wirel Commun. Networking Mob. Comput. (2007) 2555-2558.

[41] Z. Shan, T.-P. Yum, Precise localization with smart antennas in adhoc networks, GLOBECOM IEEE Global Telecommun. Conf. (2007) 1053-1057.

[42] N. Bulusu, J. Heidemann, D. Estrin, GPS-less low-cost outdoor localization for very small devices, IEEE Pers. Commun. 7 (2000) 28-34.

[43] G.Q. Gao, L. Lei, An improved node localization algorithm based on DV-HOP in WSN, Proc. IEEE Int. Conf. Adv. Comput. Control, ICACC. 4 (2010) 321-324.

[44] L. Doherty, K.S.J. Pister, L. El Ghaoui, Convex position estimation in wireless sensor networks, Proc. IEEE INFOCOM 3 (2001) 1655-1663.
[45] Y. Zhou, X. Ao, S. Xia, An improved APIT node self-localization algorithm in WSN, in: Proc. World Congr. Intelligent Control Autom. WCICA., 2008, pp. 7576-7581.

[46] J. Blumenthal, R. Grossmann, F. Golatowski, D. Timmermann, Weighted centroid localization in Zigbee-based sensor networks, IEEE Int. Symp. Intelligent Signal Process., WISP. (2007).

[47] S. Schuhmann, K. Herrmann, K. Rothermel, J. Blumenthal, D. Timmermann, Improved weighted centroid localization in smart ubiquitous environments, Lect. Notes Comput. Sci. 5061 LNCS (2008) 20-34.

[48] R. Behnke, D. Timmermann, AWCL: Adaptive weighted centroid localization as an efficient improvement of coarse grained localization, in: Workshop Positioning, Navig. Commun., WPNC. 2008, pp. 243-250.

[49] R. Behnke, J. Salzmann, R. Grobßmann, D. Lieckfeldt, D. Timmermann, K. Thurow, Strategies to overcome border area effects of coarse grained localization, in: Proc. - Workshop Positioning, Navig. Commun., WPNC., 2009, pp. 95-102.

[50] H. Lee, J.L. Welch, N.H. Vaidya, Location tracking using quorums in mobile ad hoc networks, Ad Hoc Networks 1 (2003) 371-381.

[51] H. Chu, R. Jan, A GPS-less, outdoor, self-positioning method for wireless sensor networks, Ad Hoc Networks 5 (2007) 547-557.

[52] C. Wang, K. Liu, N. Xiao, A range free localization algorithm based on restricted-area for wireless sensor networks, in: Proc. - Int. MultiConf. Comput. Global Inf. Technol., ICCGI Conjunction ComP2P: Int. Workshop Comput. P2P Netw.: Theory Prac., 2008, pp. 97-101.

[53] C. You, P. Huang, H. Chu, Y. Chen, J. Chiang, S. Lau, Impact of sensorenhanced mobility prediction on the design of energy-efficient localization, Ad Hoc Networks 6 (2008) 1221-1237.

[54] Y. Zou, K. Chakrabarty, Target localization based on energy considerations in distributed sensor networks, Ad Hoc Networks 1 (2003) 261-272.

[55] S. Rajaee, S.M.T. Almodarresi, M.H. Sadeghi, M. Aghabozorgi, Energy efficient localization in wireless ad-hoc sensor networks using probabilistic neural network and independent component analysis, Int. Symp. Telecommun., IST. (2008) 365-370.

[56] F. Xiufang, G. Zhanqiang, Y. Mian, X. Shibo, Fuzzy distance measuring based on RSSI in wireless sensor network, in: Proc. Int. Conf. Intell. Syst. Knowl. Eng., ISKE., 2008, pp. 395-400.

[57] S. Chiang, J. Wang, Localization in wireless sensor networks by fuzzy logic system, Lect. Notes Comput. Sci. 5712 LNAI (2009) 721-728.

[58] T.I. Kvaksrud, Range Measurements in an Open Field Environment, Design Note DN018 - SWRA169A, Texas Intrument. (2008) 1-14. 